\title{
Hydrogeological Studies for Groundwater Resource and its Vulnerability to Contamination in Owo, Southwestern Nigeria
}

\author{
O.O. Falowo ${ }^{1}$ E.G. Imeokparia ${ }^{2}$ \\ ${ }^{1,2}$ MDepartment of Geology, Faculty of Physical Sciences, University of Benin, Edo State Nigeria
}

\begin{abstract}
Hydrogeological investigations were conducted at Rufus Giwa Polytechnic, Owo, Southwestern, Nigeria with the aim of evaluating the groundwater resource and vulnerability of the aquifers to contamination in the area. The geophysical survey involved Vertical Electrical Sounding (VES). The hydro-geological survey includes measurements of thickness of the vadose water, static water level determination, and hydraulic head in existing wells across the study area. A total of twenty seven (27) traverses were established in the studied area. The geoelectric section revealed 3 to 5 major layers comprising the topsoil, clay, laterite, weathered layer, partly weathered layer/fractured basement, and fresh basement rock. The Iso-resistivity map and isopach maps shows that the northeastern and southwestern parts of the institution show a high overburden thickness suggesting a high degree of weathering. Transverse unit resistance map shows that the northwest through the central to the southeastern part of the study area constitute the medium groundwater development areas, while the northeastern and southwestern parts are high groundwater development areas. The longitudinal unit conductance map shows low - high L.U.C. values observed around southwestern and northeastern/eastern part of the area. The overburden protective capacity map shows a moderate protective at the southwestern and northeastern/eastern parts, while all other areas have poor/weak protective capacity. The static water level, water column and depth to water level vary between $316.9 m$ and $330.5 \mathrm{~m}, 1.3 \mathrm{~m}$ and $4.8 \mathrm{~m}$, and $8.1 \mathrm{~m}$ and $10.5 \mathrm{~m}$ respectively. The average depth to the water level is about $9.4 \mathrm{~m}$. This implies that the vadose zone is fairly thick; hence less vulnerable to contamination. Therefore considering the thickness of the vadose zone across the area, the resistivity parameters of the topsoil and the longitudinal conductance of the geomaterials; the southwestern and northeastern/eastern parts have would be fairly protected from contaminants arising from anthropogenic activities (moderate protective capacity) while other areas have poor/weak protective capacity.
\end{abstract}

Keywords: hydrogeology, contamination, groundwater, vulnerability

\section{Introduction}

It has been reported that at least 1.1 billion people across the world lack access to safe, clean drinking water. Nigeria with a population of over 120 million people have invested heavily in borehole Projects throughout the Country to satisfy the fast growing demand for safe water and to improve the socioeconomic development of its populace especially in the rural areas. The tasks of achieving the Millennium Development Goals (MDGs) in the area of groundwater exploitation therefore present an enormous challenge to the Country and the international community.

Despite huge investment by the government in the area of water supply, there is still severe scarcity of water supply for domestic, agricultural and industrial purposes. In addition, most of the drilled boreholes are failing at a very alarming rate. Some of the reasons for this failure rates could be due to technical, political and financial constraints. In order to meet the MDGs especially in the area of water supply, there is the need to adopt and develop mechanisms or strategies to improve construction quality and functionality of borehole projects in the research environment and in Nigeria as a whole.

Groundwater occurrence in the crystalline basement terrain of Nigeria can be very irregular due to abrupt discontinuity in lithology, thickness, and electrical properties of the overburden and weathered bedrock [9], [15]. However, the groundwater obtained from basement complex area is generally known to be of good quality [8]. Consequently, groundwater exploration within such geologic setting requires integration of geophysical data types to effectively characterize the hydrogeologic zones and to enhance successful identification of well locations.

\subsection{Description of the Project Environment}

The study area is Rufus Giwa Polytechnic, Owo, Ondo State "Fig. 1", which is within the south western part of Nigeria. It lies within longitudes $6^{\circ} 00^{1} \mathrm{E}$ and $5^{\circ} 30^{1} \mathrm{E}$ and latitudes $7^{\circ}$ $30^{1} \mathrm{~N}$ and $7^{\circ} 00^{1} \mathrm{~N}$. The study area is easily accessible by roads like Ikare - Owo highway, Benin - Ifon highway and Akure - Owo highway. 
International Journal of Science and Research (IJSR)

ISSN (Online): 2319-7064

Index Copernicus Value (2013): 6.14 | Impact Factor (2014): 5.611

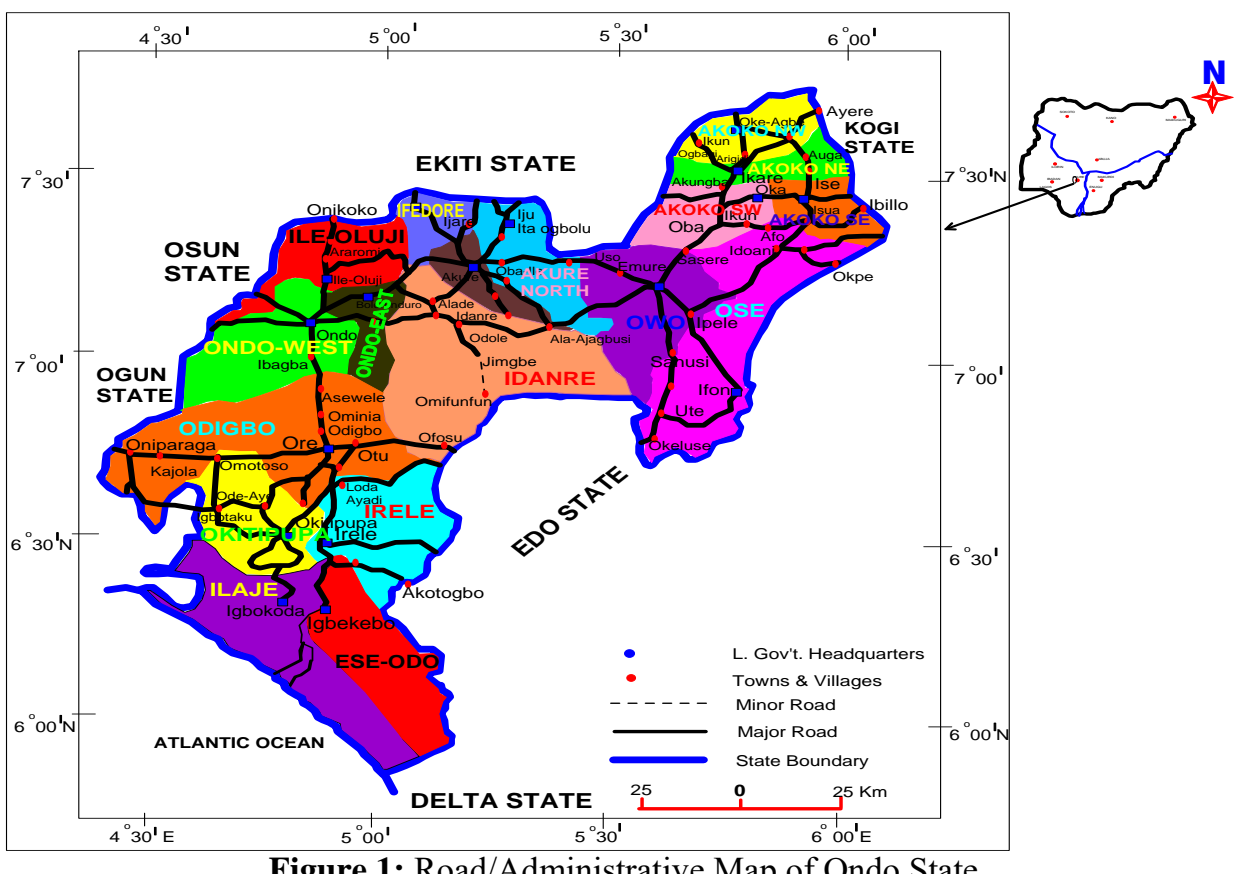

Figure 1: Road/Administrative Map of Ondo State

Owo is relatively flat, as the terrain ranges from $940 \mathrm{ft}$ to $1100 \mathrm{ft}$. However, the study area has a gently undulating topography as it ranges from $311 \mathrm{~m}$ to $342 \mathrm{~m}$ above the sea level. The area lies geographically within the tropical rain forest belt of hot and wet equatorial climatic region characterized by alternating wet and dry climate seasons [5], which is strongly controlled by seasonal fluctuation in the rate of evaporation.

The available rain data shows that mean annual rainfall ranges from $1000 \mathrm{~mm}-1500 \mathrm{~mm}$ and mean temperature of $24^{\circ} \mathrm{C}$ to $27^{\circ} \mathrm{C}$. There is rapid rainfall during the month of March and cessation during the month of November. June and September are the critical month when rainfall is usually on the high side. The vegetation is of tropical rainforest and is characterized by thick forest of broad-leaved trees that is ever green. The vegetation of the area (especially in undeveloped areas) is dense and made up of palm trees, kolanut trees and cocoa trees. Part of this area is also made the school farm.

\subsection{Geology of the Studied Area}

The area of study falls within the Southwestern basement rock, which is part of Nigerian Basement complex. The area is underlain mainly by rocks of the Migmatite - Gneiss Complex with is predominated by quartzite, granite and granite gneiss "Fig. 2". Quartzite is the most widespread rock in the area; which mineralogically contains quartz dominating mineral, other minerals such as muscovite, tremolite, microcline and biotite are common as well. Quartzites which are prominent as ridge vary in texture from massive to schistocity due to the presence of flaky minerals like mica.

\section{Method of Study}

Twenty Seven traverses were established in the investigated area. Seventy five (75) sounding stations were occupied at the study area. The location of each sounding stations in both geographic and Universal Traverse Mercator (UTM) coordinates was recorded with the aid of the GARMIN 12 channel personal navigator - Geographic Positioning System (GPS) - unit. The instrument used for the resistivity data collection was the Ohmega Resistivity meter. The current electrode spacing $(\mathrm{AB} / 2)$ was varied from $1 \mathrm{~m}$ to $65 \mathrm{~m}$ and 1 $\mathrm{m}$ to $225 \mathrm{~m}$. The metal electrodes were driven into the ground with hammers to ensure good contact with the ground. The measurements were taken at all stations with inline arrangement of the electrode with respect to the traverse lines and the apparent resistivity values were calculated.

The apparent resistivity measurements at each station were plotted against electrode spacing $(\mathrm{AB} / 2)$ on bi-logarithmic graph sheets. The resulting curves were then inspected visually to determine the nature of the subsurface layering. In each way, each curve was characterized depending upon the number and nature of the subsurface layers. Partial curve matching [13] was carried out for the quantitative interpretation of the curves.

The results of the curve matching (layer resistivities and thicknesses) were fed into the computer as starting model parameter in an iterative forward modeling technique using RESIST version 1.0 [16]. From the interpretation results, geoelectric sections along the traverses were produced. The interpreted result was considered satisfactory where a good fit of RMS between the field curves and computer generated curves is generally less than $10 \%$; as it ranges between $1.8-$ 10.7. The results were also used to generate layer parameter histograms.

Also three hand dug wells were logged to reveal estimation of depth to the water level, depth to the bedrock and water table elevation. The surface elevation at different points vary due to topographic variation, the true water level were obtained by subtracting the measured depth to the water

\section{Volume 4 Issue 12, December 2015}




\section{International Journal of Science and Research (IJSR) \\ ISSN (Online): 2319-7064}

Index Copernicus Value (2013): 6.14 | Impact Factor (2014): 5.611
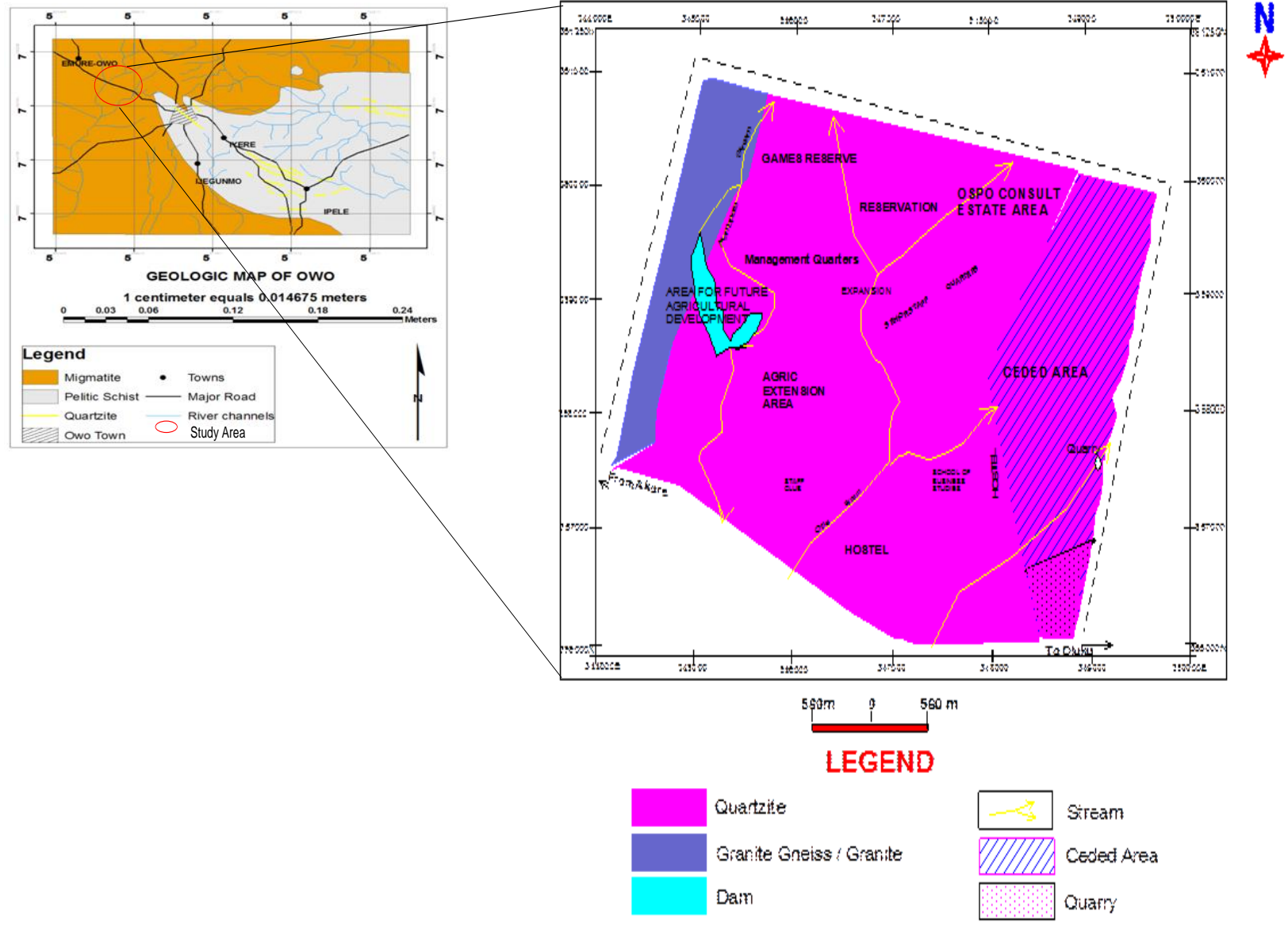

Figure 2: Geology of the Study Area

level in the hand-dug wells from the surface elevation to get uniform water levels otherwise known as the elevation of the water level [3].

$$
\text { Mathematically, } S_{w l}=E-D_{w l}
$$

Where: $S_{w l}$ is the true or uniform water level otherwise known as the static water level in the case of unconfined aquifer

$E$ is the surface elevation with respect to the mean sea level $D_{w l}$ is the depth from the surface of the earth to the water level (Well Head) in the hand-dug wells (Direct borehole logging)

The water column was calculated from the static water level data gathered from the wells using the formula;

$$
W C=T D-S_{w l}
$$

Where, WC is the Water Column (m)

TD is the Total Depth of the well

\section{Results and Discussion}

\subsection{Vertical Electrical Sounding (VES) and Field Curve}

Table 1 gives a summary of the results of the VES curves obtained from the study area. The number of layers varies between 3-layers and six-layers. Nine curve types have been identified in the study area: KH, HKH, H, KHKH, QH, HK, $\mathrm{KQ}$, $\mathrm{AKQ}$, and $\mathrm{KQH}$. The most occurring curve types identified are KH and HKH. The root mean square (RMS) error of the generated curves ranges between 1.8 and 10.7; this shows models of well smoothened, iterated curves [2]. The type curves can be classified into four distinct classes as follows:

Class 1: KQ and AKQ

Class 2: $\mathrm{H}$ and $\mathrm{QH}$

Class 3: $\mathrm{KH}, \mathrm{KQH}$, and $\mathrm{KHKH}$

Class 4: $\mathrm{HK}$ and $\mathrm{HKH}$

Examples of the Class 1 type curve (KQ and AKQ) are illustrated in "Fig. 3". The class represents a subsurface condition in which there is a highly resistive hard pan underlines a low resistive clayey soil, and the weathered layer underlines the former. The weathered layer overlies the partly weathered/fracture basement rock depending on this layer resistivity. This group of curves account for just 3.9\% of the total.

The VES curves of Class $2(\mathrm{H}$ and $\mathrm{QH})$ are characteristics of the Basement Complex saprolite produced from in-situ weathering. Examples of the sounding curves are shown in "Fig. 4". In this profile, the upper horizons, when not leached, are usually clayey and the main aquifer zone is found at the base where selective mineral decomposition has produced a gravel-like material [6]. Immediately underlying the usually low resistivity, high porosity, and low permeability weathered zone is the fresh basement. This classic architecture of the profile produces an H-type curve

Table 1: Summary of VES results obtained in the study area

\begin{tabular}{|l|l|l|l|l|l|}
\hline Traverse & VES & RESISTIVITY (Ohmns-meter) & THICKNESS $(m)$ & DEPTH $(m)$ \\
\hline
\end{tabular}




\section{International Journal of Science and Research (IJSR) \\ ISSN (Online): 2319-7064}

Index Copernicus Value (2013): 6.14 | Impact Factor (2014): 5.611

\begin{tabular}{|c|c|c|c|c|c|c|c|c|c|c|c|c|c|c|c|c|c|c|}
\hline No. & $N O$. & $P_{1}$ & $P_{2}$ & $\rho_{3}$ & $\rho_{4}$ & $\rho_{5}$ & $\rho_{6}$ & $h_{1}$ & $h_{2}$ & $h_{9}$ & $h_{4}$ & $h_{5}$ & $d_{1}$ & $d_{2}$ & $d_{3}$ & $d_{4}$ & $d_{5}$ & Type \\
\hline \multirow[t]{5}{*}{1} & 1 & 203 & 123 & 598 & 491 & & & 2.5 & \begin{tabular}{|l|l|}
4.0 \\
\end{tabular} & 1.3 & & & 2.5 & \begin{tabular}{|l|}
6.5 \\
\end{tabular} & 7.8 & & & HK \\
\hline & 2 & 341 & 189 & 145 & 499 & & & 1.0 & $\mid 14.3$ & 12.2 & & & 1.0 & 15.3 & 27.5 & & & $\mathrm{QH}$ \\
\hline & 3 & 594 & 107 & 469 & 92 & & & 0.8 & \begin{tabular}{|l|l|}
2.0 \\
\end{tabular} & 3.4 & & & 0.8 & \begin{tabular}{|l|l|}
2.8 \\
\end{tabular} & 6.2 & & & $\mathrm{HK}$ \\
\hline & 4 & 840 & 114 & 671 & 35 & 5899 & & $\begin{array}{l}0.9 \\
\end{array}$ & \begin{tabular}{|l|}
1.0 \\
\end{tabular} & 5.2 & 11.4 & & \begin{tabular}{|l|}
0.9 \\
\end{tabular} & \begin{tabular}{|l|}
1.9 \\
\end{tabular} & 7.1 & \begin{tabular}{|l|l|}
18.5 \\
\end{tabular} & & HKH \\
\hline & 5 & 814 & 157 & 48 & 10176 & & & 2.2 & 43.8 & 3.2 & & & 2.2 & 46 & 49 & & & $\mathrm{QH}$ \\
\hline \multirow[t]{2}{*}{2} & 6 & 199 & 575 & 52 & \begin{tabular}{|l|l|}
3061 \\
\end{tabular} & 10 & 26570 & 0.9 & \begin{tabular}{|l|}
1.9 \\
\end{tabular} & 3.7 & 1.9 & 3.0 & 0.9 & \begin{tabular}{|l|}
2.8 \\
\end{tabular} & 6.5 & 8.4 & 11.4 & KHKH \\
\hline & 7 & 1644 & 283 & 136 & 661 & & & $\begin{array}{l}1.1 \\
\end{array}$ & \begin{tabular}{|l|l|}
4.4 \\
\end{tabular} & 8.1 & & & \begin{tabular}{|l|l|}
1.1 \\
\end{tabular} & \begin{tabular}{|l|}
5.5 \\
\end{tabular} & $\begin{array}{ll}13.6 \\
\end{array}$ & & & $\mathrm{QH}$ \\
\hline \multirow[t]{3}{*}{3} & 8 & 237 & 176 & 322 & 71 & 678 & & $\begin{array}{l}0.8 \\
\end{array}$ & \begin{tabular}{|l|}
1.5 \\
\end{tabular} & 22 & 10.7 & & \begin{tabular}{|l|l|}
0.8 \\
\end{tabular} & \begin{tabular}{|l|}
2.3 \\
\end{tabular} & 24.3 & \begin{tabular}{|l|}
35.1 \\
\end{tabular} & & HKH \\
\hline & 9 & 1210 & 45 & 416 & & & & 1.2 & 13.5 & 14.7 & & & 1.2 & \begin{tabular}{|l}
14.7 \\
\end{tabular} & & & & $\mathrm{H}$ \\
\hline & 10 & 1462 & 244 & 12528 & & & & $\begin{array}{l}1.9 \\
\end{array}$ & 11.5 & & & & \begin{tabular}{|l|}
1.9 \\
\end{tabular} & \begin{tabular}{|l|l|}
13.4 \\
\end{tabular} & & & & $\mathrm{H}$ \\
\hline 4 & 11 & 785 & 6048 & 108 & 111179 & & & $\begin{array}{l}1.6 \\
\end{array}$ & \begin{tabular}{|l|}
1.7 \\
\end{tabular} & 6.5 & & & \begin{tabular}{|l|l|}
1.6 \\
\end{tabular} & \begin{tabular}{|l|}
3.4 \\
\end{tabular} & 9.9 & & & $\mathrm{KH}$ \\
\hline \multirow[t]{4}{*}{5} & 12 & 778 & 1633 & 49 & 10372 & & & 1.5 & \begin{tabular}{|l|l}
13.3 \\
\end{tabular} & 18.2 & & & 1.5 & \begin{tabular}{|l|}
14.8 \\
\end{tabular} & 33.0 & & & $\mathrm{KH}$ \\
\hline & 13 & 3395 & 11389 & 7814 & \begin{tabular}{|l|}
204 \\
\end{tabular} & & & $\begin{array}{l}0.3 \\
\end{array}$ & \begin{tabular}{|l|}
3.2 \\
\end{tabular} & \begin{tabular}{ll|}
10.9 \\
\end{tabular} & & & \begin{tabular}{|l|}
0.3 \\
\end{tabular} & \begin{tabular}{|l|}
3.6 \\
\end{tabular} & 14.5 & & & KQ \\
\hline & 14 & 253 & 1208 & 253 & 27561 & & & $\begin{array}{l}0.6 \\
\end{array}$ & \begin{tabular}{|l|l|}
7.0 \\
\end{tabular} & 55.3 & & & \begin{tabular}{|l|l|}
0.6 \\
\end{tabular} & \begin{tabular}{|l|}
7.6 \\
\end{tabular} & 62.9 & & & $\mathrm{KH}$ \\
\hline & 15 & 1464 & 2029 & 13798 & 680 & 158 & & 19.8 & \begin{tabular}{|l|}
1.2 \\
\end{tabular} & 22.0 & 1.9 & & 19.8 & 20.9 & 43.0 & 44.9 & & AKQ \\
\hline \multirow[t]{3}{*}{6} & 16 & 895 & 378 & 633 & 226 & 542 & & 1.0 & \begin{tabular}{|l|l|}
2.6 \\
\end{tabular} & 5.5 & $\begin{array}{l}9.2 \\
\end{array}$ & & 1.0 & \begin{tabular}{|l|}
3.6 \\
\end{tabular} & 9.1 & \begin{tabular}{|l|l|}
18.3 \\
\end{tabular} & & $\mathrm{HKH}$ \\
\hline & $\begin{array}{ll}17 \\
\end{array}$ & 110 & 164 & 58 & 167 & & & 1.0 & \begin{tabular}{|l|l}
13.3 \\
\end{tabular} & 20.5 & & & 1.0 & \begin{tabular}{|l|}
14.2 \\
\end{tabular} & 34.8 & & & $\mathrm{KH}$ \\
\hline & 18 & 46 & 264 & 47 & 12325 & & & $\begin{array}{l}1.0 \\
\end{array}$ & \begin{tabular}{|l|l|}
6.9 \\
\end{tabular} & 14.6 & & & \begin{tabular}{|l|l}
1.0 \\
\end{tabular} & \begin{tabular}{|l|}
7.9 \\
\end{tabular} & 22.5 & & & $\mathrm{KH}$ \\
\hline \multirow[t]{3}{*}{7} & 19 & 667 & 353 & 852 & 244 & 7097 & & 1.0 & \begin{tabular}{|l|}
1.3 \\
\end{tabular} & 17.9 & 88.8 & & 1.0 & \begin{tabular}{|l|}
2.3 \\
\end{tabular} & 20.2 & \begin{tabular}{|l|l|}
109 \\
\end{tabular} & & HKH \\
\hline & 20 & 180 & 31 & 3858 & 12 & 652 & & 1.4 & \begin{tabular}{|l|}
2.0 \\
\end{tabular} & 1.5 & 3.3 & & 1.4 & \begin{tabular}{|l|}
3.3 \\
\end{tabular} & 4.8 & \begin{tabular}{|l|l|}
8.1 \\
\end{tabular} & & HKH \\
\hline & 21 & 415 & 219 & 857 & 222 & 5432 & & 0.7 & \begin{tabular}{|l|}
1.2 \\
\end{tabular} & 4.0 & 65.0 & & 0.7 & \begin{tabular}{|l|}
1.9 \\
\end{tabular} & 5.9 & \begin{tabular}{|l|}
70.9 \\
\end{tabular} & & HKH \\
\hline \multirow[t]{3}{*}{8} & 22 & 171 & 719 & 141 & 1406 & 40 & \begin{tabular}{|l|}
376 \\
\end{tabular} & 0.5 & \begin{tabular}{|l|l|}
1.0 \\
\end{tabular} & 3.0 & 2.3 & \begin{tabular}{|l|l|}
9.1 \\
\end{tabular} & 0.5 & \begin{tabular}{|l|}
1.5 \\
\end{tabular} & 4.5 & \begin{tabular}{|l|l|}
6.8 \\
\end{tabular} & 15.9 & KHKH \\
\hline & 23 & 219 & 1474 & 60 & 562 & 84 & \begin{tabular}{|l|}
327 \\
\end{tabular} & 0.4 & \begin{tabular}{|l|l|}
0.4 \\
\end{tabular} & 1.1 & 8.2 & \begin{tabular}{|l|l|}
8.3 \\
\end{tabular} & \begin{tabular}{|l|l|}
0.4 \\
\end{tabular} & \begin{tabular}{|l|}
0.8 \\
\end{tabular} & 1.9 & \begin{tabular}{|l|l|}
10.1 \\
\end{tabular} & 18.4 & KHKH \\
\hline & 24 & 270 & 64 & 522 & & & & 3.9 & 26.9 & & & & 3.9 & 30.8 & & & & $\mathrm{H}$ \\
\hline 9 & 25 & 254 & 535 & 131 & 44 & 955 & & 0.9 & \begin{tabular}{|l|l|}
1.0 \\
\end{tabular} & 12.7 & 38.6 & & \begin{tabular}{|l|}
0.9 \\
\end{tabular} & \begin{tabular}{|l|}
1.9 \\
\end{tabular} & 14.6 & 53.2 & & $\mathrm{KQH}$ \\
\hline & 26 & 200 & 3039 & 141 & 361 & & & 0.5 & \begin{tabular}{|l|}
0.3 \\
\end{tabular} & 41.7 & & & $\begin{array}{ll}0.5 \\
\end{array}$ & \begin{tabular}{|l|}
0.8 \\
\end{tabular} & 42.4 & & & $\mathrm{KH}$ \\
\hline & 27 & 286 & \begin{tabular}{|l|}
716 \\
\end{tabular} & 18 & 1579 & & & $\begin{array}{l}1.1 \\
\end{array}$ & \begin{tabular}{|l|}
2.9 \\
\end{tabular} & 6.0 & & & 1.1 & \begin{tabular}{|l|}
4.0 \\
\end{tabular} & 10.0 & & & $\mathrm{KH}$ \\
\hline 10 & 28 & 331 & 50 & 229 & 16 & 2498 & & 1.3 & \begin{tabular}{|l|}
1.3 \\
\end{tabular} & 3.1 & 7.6 & & 1.3 & \begin{tabular}{|l|}
2.6 \\
\end{tabular} & 5.8 & \begin{tabular}{|l|l|}
13.4 \\
\end{tabular} & & HKH \\
\hline & 29 & 82 & 726 & 46 & 284 & & & 0.5 & \begin{tabular}{|l|}
1.3 \\
\end{tabular} & 24.5 & & & 0.5 & $\begin{array}{ll}1.9 \\
\end{array}$ & 26.4 & & & $\mathrm{KH}$ \\
\hline 11 & 30 & 120 & 441 & 20 & 301 & 52 & 46638 & $\begin{array}{l}0.9 \\
\end{array}$ & \begin{tabular}{|l|}
1.2 \\
\end{tabular} & 1.5 & 8.7 & 22.8 & $\begin{array}{l}0.9 \\
\end{array}$ & \begin{tabular}{|l|}
2.0 \\
\end{tabular} & 3.5 & \begin{tabular}{|l|}
12.2 \\
\end{tabular} & 35.0 & KHKH \\
\hline 12 & 31 & 516 & 83 & 14979 & 19 & 3941 & & 2.5 & \begin{tabular}{|l|}
2.8 \\
\end{tabular} & 2.3 & 20.3 & & 2.5 & \begin{tabular}{|l|}
5.3 \\
\end{tabular} & 7.5 & 27.8 & & HKH \\
\hline & 32 & 65 & 1258 & 20 & \begin{tabular}{|l}
14478 \\
\end{tabular} & & & 0.4 & \begin{tabular}{|l|}
1.0 \\
\end{tabular} & 6.3 & & & \begin{tabular}{|l|}
0.4 \\
\end{tabular} & \begin{tabular}{|l|}
1.4 \\
\end{tabular} & 7.8 & & & $\mathrm{KH}$ \\
\hline 13 & 33 & 515 & \begin{tabular}{|l|l|}
587 \\
\end{tabular} & 104 & 430 & & & $\begin{array}{l}1.0 \\
\end{array}$ & \begin{tabular}{|l|l|}
7.4 \\
\end{tabular} & 47.4 & & & \begin{tabular}{|l|l}
1.0 \\
\end{tabular} & \begin{tabular}{|l|l|}
8.4 \\
\end{tabular} & \begin{tabular}{|l}
55.9 \\
\end{tabular} & & & $\mathrm{KH}$ \\
\hline 14 & 34 & 262 & 353 & 69 & 849 & & & $\begin{array}{l}0.8 \\
\end{array}$ & \begin{tabular}{|l|}
9.5 \\
\end{tabular} & 15.5 & & & \begin{tabular}{|l|l|}
0.8 \\
\end{tabular} & \begin{tabular}{|l|}
10.3 \\
\end{tabular} & 25.8 & & & $\mathrm{KH}$ \\
\hline & 35 & 135 & 39 & 477 & 24 & 1280 & & $\begin{array}{l}0.9 \\
\end{array}$ & \begin{tabular}{|l|}
1.0 \\
\end{tabular} & 2.6 & 13.8 & & \begin{tabular}{|l|}
0.9 \\
\end{tabular} & \begin{tabular}{|l|}
1.9 \\
\end{tabular} & 4.5 & \begin{tabular}{|l|}
18.3 \\
\end{tabular} & & HKH \\
\hline 15 & 36 & 183 & 41 & 338 & & & & 1.0 & \begin{tabular}{|l|}
8.0 \\
\end{tabular} & & & & 1.0 & \begin{tabular}{|l|}
9.0 \\
\end{tabular} & & & & $\mathrm{H}$ \\
\hline & 37 & 658 & 448 & 206 & 986 & & & 0.9 & \begin{tabular}{|l|}
3.3 \\
\end{tabular} & 7.7 & & & 0.9 & \begin{tabular}{|l|l|}
4.3 \\
\end{tabular} & 11.9 & & & $\mathrm{QH}$ \\
\hline 16 & 38 & 332 & \begin{tabular}{|l|l}
492 \\
\end{tabular} & 104 & \begin{tabular}{|l|}
2289 \\
\end{tabular} & & & $\begin{array}{l}0.9 \\
\end{array}$ & \begin{tabular}{|l|}
2.9 \\
\end{tabular} & 64.5 & & & \begin{tabular}{|l|}
0.9 \\
\end{tabular} & \begin{tabular}{|l|}
3.8 \\
\end{tabular} & 68.3 & & & $\mathrm{KH}$ \\
\hline & 39 & 328 & 211 & 256 & 71 & 9122 & & 1.8 & 13.4 & 21.2 & 54.4 & & 1.8 & 13.4 & 21.2 & \begin{tabular}{|l|l|}
54.4 \\
\end{tabular} & & $\mathrm{HKH}$ \\
\hline & 40 & 396 & 472 & 114 & 678 & & & 0.9 & \begin{tabular}{|l|}
3.1 \\
\end{tabular} & 87.3 & & & $\begin{array}{ll}0.9 \\
\end{array}$ & \begin{tabular}{|l|}
4.0 \\
\end{tabular} & 91.3 & & & $\mathrm{KH}$ \\
\hline 17 & 41 & 80 & 106 & 63 & 269 & & & $\begin{array}{l}0.7 \\
\end{array}$ & \begin{tabular}{|l|}
2.2 \\
\end{tabular} & $\begin{array}{ll}19.8 \\
\end{array}$ & & & \begin{tabular}{|l|l|}
0.7 \\
\end{tabular} & \begin{tabular}{|l|}
2.9 \\
\end{tabular} & 22.7 & & & $\mathrm{KH}$ \\
\hline & 42 & 227 & 138 & 97 & 394 & & & 1.2 & \begin{tabular}{|l|}
1.9 \\
\end{tabular} & 30. & & & 1.2 & \begin{tabular}{|l|}
3.1 \\
\end{tabular} & 33.6 & & & $\mathrm{QH}$ \\
\hline 18 & 43 & 274 & 460 & 68 & 2382 & & & 0.7 & \begin{tabular}{|l|l|} 
\\
\end{tabular} & 15.9 & & & 0.7 & \begin{tabular}{|l|}
1.5 \\
\end{tabular} & 17.4 & & & $\mathrm{KH}$ \\
\hline & 44 & 97 & 95 & 149 & 108 & 10441 & & 1.6 & \begin{tabular}{|l|}
9.3 \\
\end{tabular} & 5.9 & 28.8 & & $\begin{array}{l}1.6 \\
\end{array}$ & \begin{tabular}{|l|}
10.8 \\
\end{tabular} & 16.8 & 45.6 & & HKH \\
\hline & 45 & 118 & 337 & 48 & 772 & 41 & \begin{tabular}{|l|}
3527 \\
\end{tabular} & 0.6 & \begin{tabular}{|l|}
0.5 \\
\end{tabular} & 2.1 & 2.2 & \begin{tabular}{|l|}
21.5 \\
\end{tabular} & \begin{tabular}{|l|l|}
0.6 \\
\end{tabular} & \begin{tabular}{|l|}
1.2 \\
\end{tabular} & 3.3 & 5.5 & 27.0 & KHKH \\
\hline 19 & 46 & 304 & 790 & 165 & 535 & & & 1.0 & \begin{tabular}{|l|l|}
1.4 \\
\end{tabular} & 30.4 & & & 1.0 & 2.4 & 32.8 & & & $\mathrm{KH}$ \\
\hline & \begin{tabular}{|l|}
47 \\
\end{tabular} & 338 & 740 & 336 & 1132 & 382 & \begin{tabular}{|l|l}
757 \\
\end{tabular} & 0.9 & \begin{tabular}{|l|}
1.5 \\
\end{tabular} & 6.5 & 2.0 & \begin{tabular}{|l|l|}
41.7 \\
\end{tabular} & $\begin{array}{ll}0.9 \\
\end{array}$ & \begin{tabular}{|l|}
2.3 \\
\end{tabular} & 8.8 & 10.8 & 52.5 & KHKH \\
\hline 20 & 48 & 147 & \begin{tabular}{|l|}
297 \\
\end{tabular} & 59 & 579 & & & 1.0 & \begin{tabular}{|l|}
1.3 \\
\end{tabular} & 18.5 & & & 1.0 & \begin{tabular}{|l|}
2.3 \\
\end{tabular} & 20.8 & & & $\mathrm{KH}$ \\
\hline & \begin{tabular}{|l|}
49 \\
\end{tabular} & 243 & \begin{tabular}{|l|l|}
479 \\
\end{tabular} & 82 & 337 & & & 1.0 & \begin{tabular}{|l|}
2.7 \\
\end{tabular} & 19.2 & & & 1.0 & \begin{tabular}{|l|}
3.5 \\
\end{tabular} & 22.8 & & & $\mathrm{KH}$ \\
\hline & \begin{tabular}{|l|}
50 \\
\end{tabular} & 342 & 322 & 138 & 1362 & & & 1.3 & \begin{tabular}{|l|}
1.4 \\
\end{tabular} & 46.9 & & & 1.3 & \begin{tabular}{|l|}
2.7 \\
\end{tabular} & 49.6 & & & $\mathrm{QH}$ \\
\hline & 51 & 543 & 780 & 264 & $\mid 11207$ & & & 0.5 & \begin{tabular}{|l|}
3.9 \\
\end{tabular} & 144.2 & & & 0.5 & 4.4 & 148.6 & & & $\mathrm{KH}$ \\
\hline 21 & 52 & 369 & 259 & 93 & 177578 & & & 5.1 & \begin{tabular}{|l|l|}
2.4 \\
\end{tabular} & 29.9 & & & \begin{tabular}{|l|l|}
5.1 \\
\end{tabular} & \begin{tabular}{|l|}
7.5 \\
\end{tabular} & 37.4 & & & $\mathrm{QH}$ \\
\hline & \begin{tabular}{|l|}
53 \\
\end{tabular} & 538 & 105 & 240 & & & & 1.2 & 25.7 & & & & 1.2 & \begin{tabular}{|l|}
26.9 \\
\end{tabular} & & & & $\mathrm{H}$ \\
\hline & \begin{tabular}{|l|}
54 \\
\end{tabular} & 305 & 38 & 278 & & & & $\begin{array}{l}0.9 \\
\end{array}$ & \begin{tabular}{|l|l}
13.8 \\
\end{tabular} & & & & \begin{tabular}{|l|}
0.9 \\
\end{tabular} & \begin{tabular}{|l}
14.7 \\
\end{tabular} & & & & $\mathrm{H}$ \\
\hline 22 & 55 & 554 & 823 & 60 & \begin{tabular}{|l|l|}
3520 \\
\end{tabular} & & & 1.1 & \begin{tabular}{|l|l|}
3.0 \\
\end{tabular} & 24.8 & & & \begin{tabular}{|l|l|}
1.1 \\
\end{tabular} & \begin{tabular}{|l|l|}
4.1 \\
\end{tabular} & 28.9 & & & $\mathrm{KH}$ \\
\hline & 56 & 294 & \begin{tabular}{|l|}
183 \\
\end{tabular} & 445 & 57 & 487 & & 1.0 & \begin{tabular}{|l|}
1.3 \\
\end{tabular} & 3.2 & 21.7 & & 1.0 & \begin{tabular}{|l|}
2.3 \\
\end{tabular} & 5.5 & 27.2 & & HKH \\
\hline & \begin{tabular}{|l|}
57 \\
\end{tabular} & 727 & 28 & 1410 & & & & 1.5 & $\mid 11.5$ & & & & 1.5 & \begin{tabular}{|l|l}
13.0 \\
\end{tabular} & & & & $\mathrm{H}$ \\
\hline & 58 & 275 & 33 & 1420 & & & & 1.1 & 15.5 & & & & 1.4 & 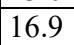 & & & & $\mathrm{H}$ \\
\hline & 59 & 117 & 202 & 40 & 1480 & & & 0.8 & \begin{tabular}{|l|}
2.8 \\
\end{tabular} & 26.9 & & & 0.8 & 3.5 & 30.4 & & & $\mathrm{KH}$ \\
\hline 23 & 60 & 227 & 652 & 134 & \begin{tabular}{|l|l}
3029 \\
\end{tabular} & & & $\begin{array}{l}1.0 \\
\end{array}$ & \begin{tabular}{|l|l|}
11.4 \\
\end{tabular} & 59.2 & & & 1.0 & \begin{tabular}{|l|}
12.4 \\
\end{tabular} & 71.7 & & & $\mathrm{KH}$ \\
\hline & $\begin{array}{ll}61 \\
\end{array}$ & 593 & 987 & 111 & 3126 & & & 1.0 & \begin{tabular}{|l|l}
19.1 \\
\end{tabular} & 52.9 & & & 1.0 & 20.1 & 73.0 & & & $\mathrm{KH}$ \\
\hline 24 & 62 & 347 & 653 & 65 & \begin{tabular}{|l|l|}
3421 \\
\end{tabular} & & & 1.1 & $\mid$\begin{tabular}{|l|}
11.1 \\
\end{tabular} & 43.9 & & & \begin{tabular}{|l|l|}
1.1 \\
\end{tabular} & \begin{tabular}{|l|l|}
12.1 \\
\end{tabular} & 56.0 & & & $\mathrm{KH}$ \\
\hline & 63 & 301 & 583 & 77 & 402 & & & 1.1 & \begin{tabular}{|l|l|}
7.1 \\
\end{tabular} & 26.8 & & & 1.1 & \begin{tabular}{|l|}
8.2 \\
\end{tabular} & 35.0 & & & $\mathrm{KH}$ \\
\hline 25 & 64 & 229 & 901 & 207 & 907 & & & 0.9 & \begin{tabular}{|l|}
2.3 \\
\end{tabular} & 28.8 & & & \begin{tabular}{|l|}
0.9 \\
\end{tabular} & \begin{tabular}{|l|}
3.2 \\
\end{tabular} & 32.1 & & & $\mathrm{KH}$ \\
\hline & 65 & 247 & 501 & 163 & 752 & & & 1.0 & \begin{tabular}{|l|}
4.7 \\
\end{tabular} & 34.1 & & & 1.0 & \begin{tabular}{|l|}
5.7 \\
\end{tabular} & 39.8 & & & КН \\
\hline
\end{tabular}

Volume 4 Issue 12, December 2015 
International Journal of Science and Research (IJSR)

ISSN (Online): 2319-7064

Index Copernicus Value (2013): 6.14 | Impact Factor (2014): 5.611

\begin{tabular}{|c|c|c|c|c|c|c|c|c|c|c|c|c|c|c|c|c|c|c|}
\hline & 66 & 302 & 618 & 252 & 914 & & & 2.8 & 9.2 & 15.6 & & & 2.8 & 12.0 & 27.6 & & & $\mathrm{KH}$ \\
\hline & 67 & 627 & 936 & 355 & 7581 & & & 1.1 & 10.6 & 43.5 & & & 1.1 & 11.7 & 55.2 & & & $\mathrm{KH}$ \\
\hline \multirow[t]{4}{*}{26} & 68 & 277 & 1050 & 558 & 1185 & 106 & 776 & 1.1 & \begin{tabular}{|l|}
0.6 \\
\end{tabular} & 5.3 & 2.1 & 24.7 & 1.1 & \begin{tabular}{|l|}
1.7 \\
\end{tabular} & 7.0 & 9.1 & 33.8 & KHKH \\
\hline & 69 & 91 & 298 & 43 & 401 & & & 1.2 & 6.6 & 18.2 & & & 1.2 & $\begin{array}{ll}7.8 \\
\end{array}$ & 26.0 & & & $\mathrm{KH}$ \\
\hline & 70 & 167 & 952 & 54 & 919 & & & 1.0 & \begin{tabular}{|l|}
0.9 \\
\end{tabular} & 30.6 & & & 1.0 & \begin{tabular}{|l}
1.9 \\
\end{tabular} & 32.5 & & & $\mathrm{KH}$ \\
\hline & 71 & 309 & 3017 & 38 & 233 & & & 0.5 & \begin{tabular}{|l|}
0.8 \\
\end{tabular} & 10.0 & & & 0.5 & \begin{tabular}{|l|}
1.3 \\
\end{tabular} & 11.2 & & & $\mathrm{KH}$ \\
\hline \multirow[t]{4}{*}{27} & 72 & 308 & 1019 & 130 & 463 & & & 1.1 & 3.6 & 39.9 & & & 1.1 & 4.7 & 44.6 & & & $\mathrm{KH}$ \\
\hline & 73 & 862 & 1223 & 119 & 383 & & & 1.0 & 1.7 & 16.4 & & & 1.0 & 2.7 & 19.1 & & & $\mathrm{KH}$ \\
\hline & 74 & 340 & 994 & 35 & 1872 & & & 1.0 & \begin{tabular}{|l|}
0.3 \\
\end{tabular} & 21.5 & & & 1.0 & \begin{tabular}{|l|}
1.2 \\
\end{tabular} & 22.8 & & & $\mathrm{KH}$ \\
\hline & 75 & 223 & 51 & 242 & & & & 1.7 & \begin{tabular}{|l|}
21.3 \\
\end{tabular} & & & & 1.7 & 23.0 & & & & $\mathrm{H}$ \\
\hline
\end{tabular}

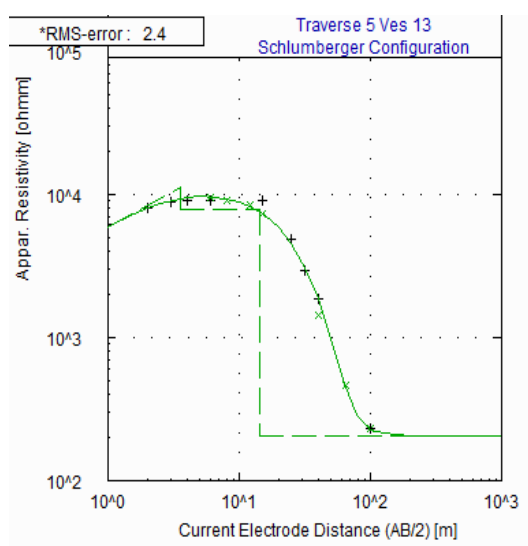

"(a)"

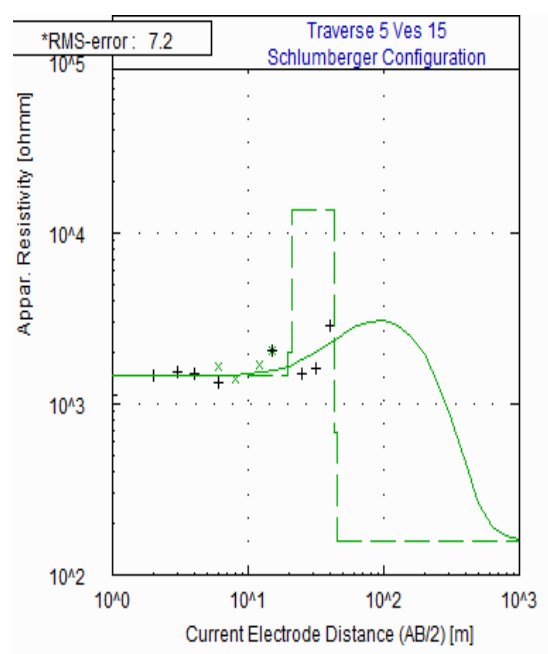

“(b)"

Figure 3: Interpretation Model of Typical Class 1 Type
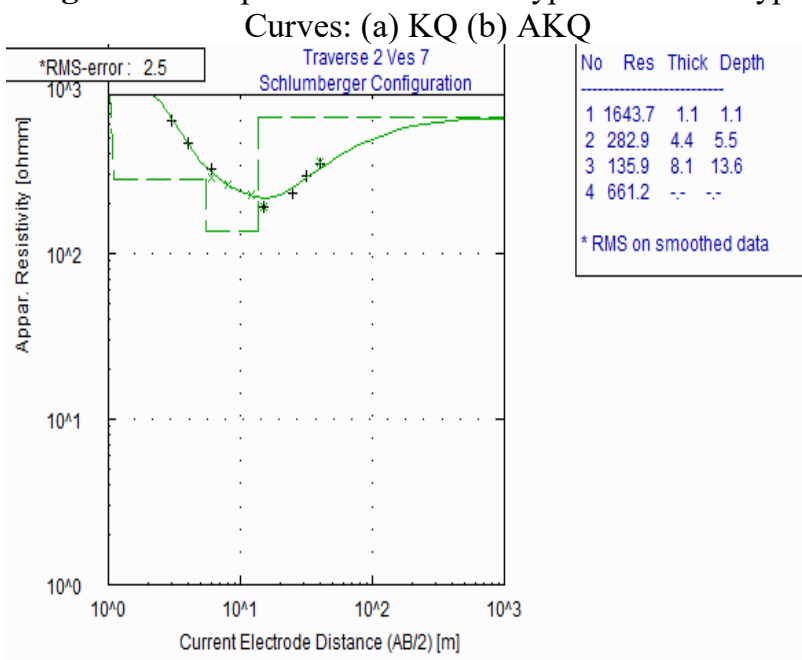

No Res Thick Depth

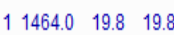

$\begin{array}{llll}2 & 2028.5 & 1.2 & 20.9 \\ 3 & 13797.5 & 22.0 & 43.0\end{array}$

$\begin{array}{llll}4 & 679.8 & 1.9 & 44.9\end{array}$

$5157.6 \quad \because-\cdots$

${ }^{*}$ RMS on smoothed data

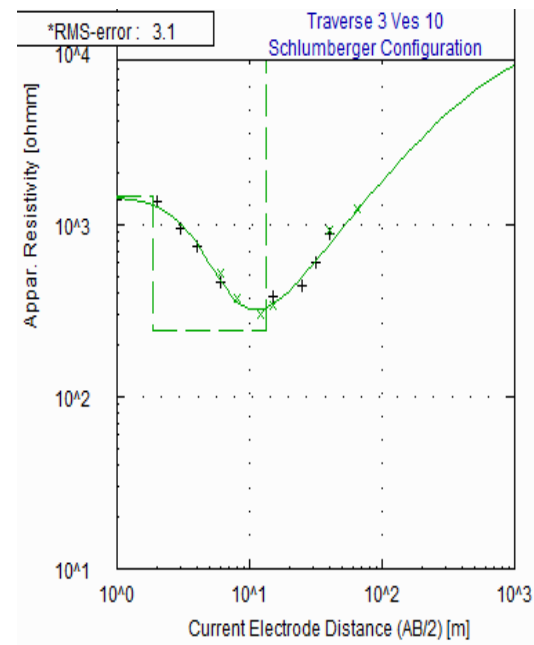

"(b)"

Figure 4: Interpretation Model of Typical Class 2 Type Curves: (a) QH (b) H

Signature, which was found to be $16 \%$ of the total curve types. In the $\mathrm{QH}$, the topsoil may be divided into two horizons with the lower one being more conductive. This may be common in areas where there is regolith (transported material) in addition to the usual in-situ saprolite. The $\mathrm{QH}$ is symptomatic of a succession in which a more conductive clayey weathered layer lies directly on the fresh basement. This curve type account for $9 \%$ of the total curve types.

Types curves of Class 3 (KH, KQH, and KHKH) "Fig. 5" which account for $47.3 \%$ of the total, are typical of a succession of relatively low and high resistivity layer. The $\mathrm{KH}$ four-layer setting which constitutes about $46.7 \%$ of the curves is found where a highly resistive lateritic hard pan underlies low resistivity clayey topsoil, and the weathered layer in turn underlies the former. The weathered layer overlies the fresh basement. In the KHKH type, the fifth layer is a confined fracture basement. The curves in Class 4 (HK and HKH) are shown in "Fig. 6". Here the succession of the subsurface layers starts with highly resistive topsoil followed by a more conductive horizon and then another less conductive layer underlines the latter, which overlies the fresh basement. HKH type is a succession of five layers i.e. the topsoil, weathered layer, fresh basement, confined fracture basement and fresh basement. The HK and $\mathrm{HKH}$ account for $2.7 \%$ and $16 \%$ of the total curve types respectively. 


\section{International Journal of Science and Research (IJSR) \\ ISSN (Online): 2319-7064}

Index Copernicus Value (2013): 6.14 | Impact Factor (2014): 5.611

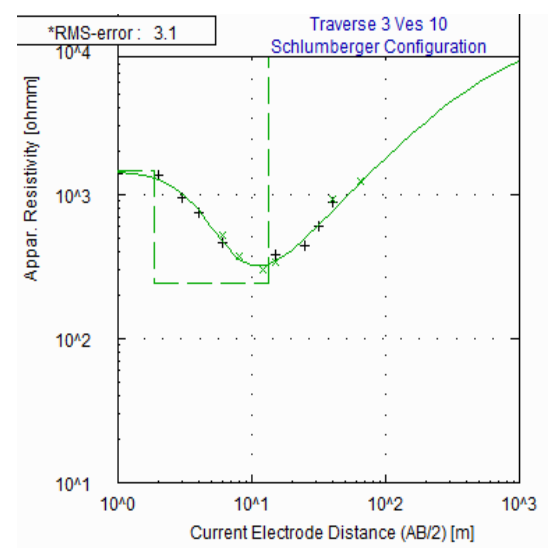

"(a)"

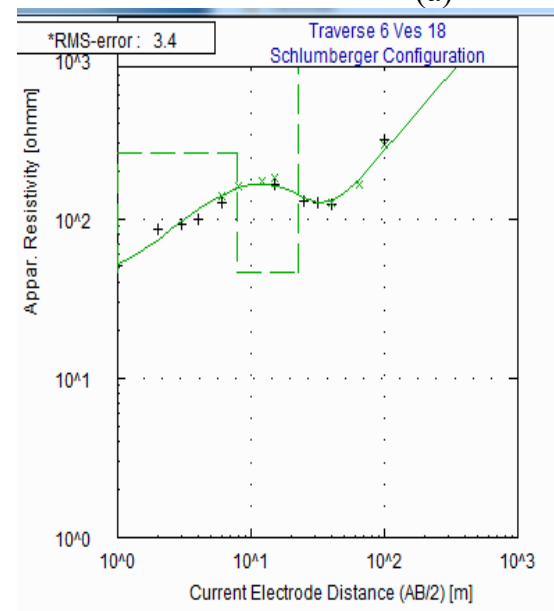

"(b)"

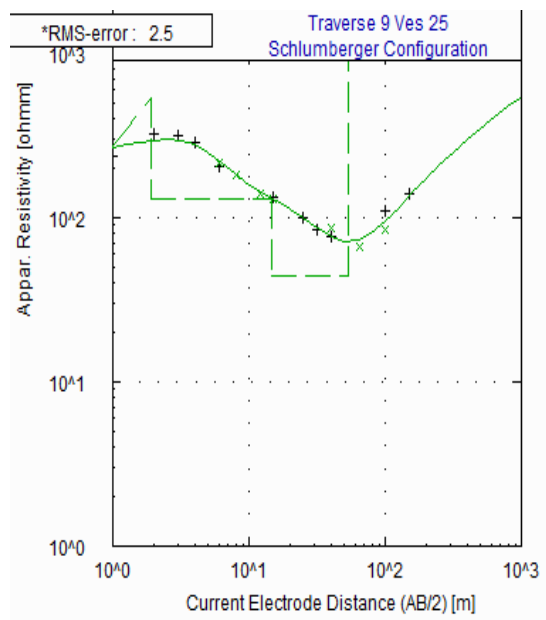

“(c)"

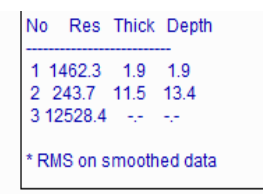

\begin{tabular}{|lccc|}
\hline No & Res & Thick Depth \\
\hline 1 & 46.0 & 1.0 & 1.0 \\
2 & 263.8 & 6.9 & 7.9 \\
3 & 46.9 & 14.6 & 22.5 \\
4 & 12314.5 & -- & $-\cdot$ \\
* & & & \\
*MS on smoothed data
\end{tabular}

No Res Thick Depth

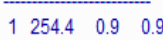

$\begin{array}{llll}2 & 534.6 & 1.0 & 1.9\end{array}$

$\begin{array}{lllll}3 & 130.6 & 12.7 & 14.6 \\ 4 & 44.2 & 38.6 & 53.2\end{array}$

$50549 \quad \cdots$

* RMS on smoothed data

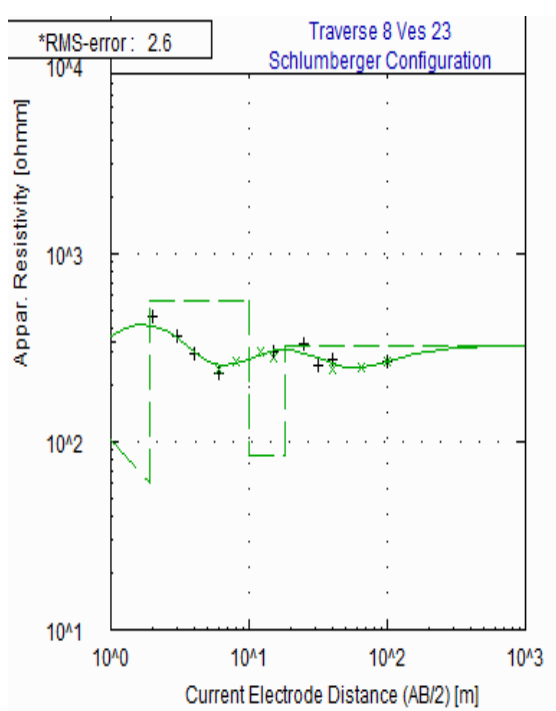

"(d)"

Figure 5: Interpretation Model of Typical Class 3 Type Curves: (a) H (b) KH (c) KQH (d) KHKH

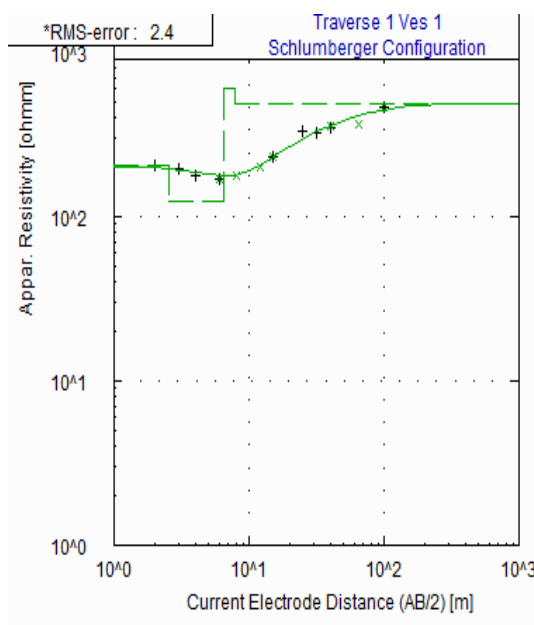

“(a)"

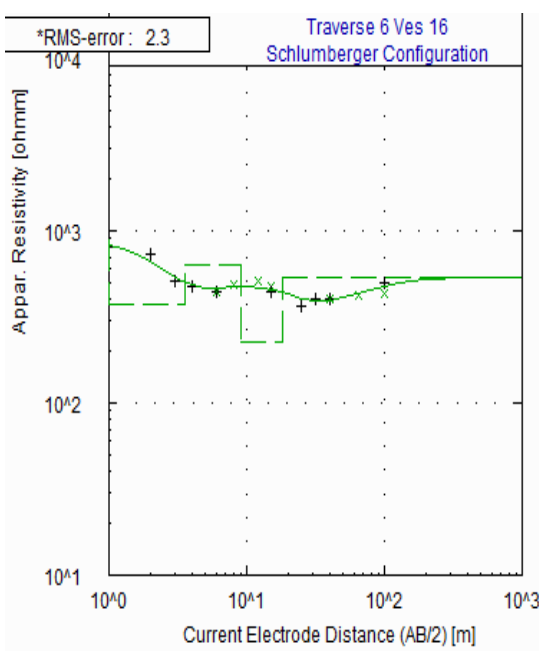

"(b)"

\begin{tabular}{|lccc} 
No & Res & Thick & Depth \\
\hline 1 & 219.0 & 0.4 & 0.4 \\
2 & 1473.7 & 0.4 & 0.8 \\
3 & 59.9 & 1.1 & 1.9 \\
4 & 562.4 & 8.2 & 10.1 \\
5 & 84.1 & 8.3 & 18.4 \\
6 & 326.6 & $-\because$ & - \\
* & & \\
RMS on smoothed data
\end{tabular}

RMS on smoothed data
No Res Thick Depth

$\begin{array}{llll}2 & 377.8 & 2.6 & 3.6\end{array}$

$\begin{array}{llll}3 & 632.7 & 5.5 & 9.1\end{array}$

$\begin{array}{lllll}5 & 541.5 & \ddots & \ddots\end{array}$

*RMS on smoothed data $\begin{array}{llll}1894.6 & 1.0 & 10\end{array}$

$\begin{array}{llll}4 & 226.1 & 9.2 & 18.3\end{array}$

Figure 6: Interpretation Model of Typical Class 4 Type Curves: (a) HK (b) HKH 


\section{International Journal of Science and Research (IJSR) \\ ISSN (Online): 2319-7064}

Index Copernicus Value (2013): 6.14 | Impact Factor (2014): 5.611

\subsection{Geoelectrical Maps}

\section{Iso-resistivity Map of the Topsoil}

Aquifer vulnerability study was done by assessing the topsoil resistivity distribution. "Fig. 7" shows the isorestivity map of the topsoil in the study area. The topsoil resistivity varies between $46 \Omega-\mathrm{m}$ and $1644 \Omega-\mathrm{m}$. The map shows that the topsoil resistivity is generally between $300 \Omega-\mathrm{m}$ and $600 \Omega$ $\mathrm{m}$ indicating clayey sand; while the southwestern part is generally ranging from $600 \Omega-\mathrm{m}$ to $900 \Omega-\mathrm{m}$ indicating a composition of clayey sand and laterite. The central part and far southeastern region of the institution are characterized by high degree of inhomogeneity.

\section{Iso-restivity Map of the Layer overlying the Weathered Layer}

The isorestivity map of the layer above the weathered layer in the study area is shown in "Fig. 8". The map shows resistivity ranging from $39 \Omega-\mathrm{m}$ to $3039 \Omega-\mathrm{m}$. There are pockets of low resistivity values observed mostly towards the southeastern part of the institution, while high resistivity values (greater than $600 \Omega-\mathrm{m}$ ) are found around the central area. However resistivity values in the range of $200 \Omega-\mathrm{m}$ to $1000 \Omega-\mathrm{m}$ is the most dominant in the study area. This implies that the layer above the weathered layer is generally clayey sand/lateritic clay in composition.

\section{Iso-resistivity Map of the Weathered Layer}

The resistivity of the weathered layer determined the degree of saturation of weathered zone. "Fig. 9" shows the resistivity distribution of the weathered layer in the study area. The resistivity ranges from 31 to $1258 \Omega-\mathrm{m}$. The map shows high degree of variation in resistivity values in the area. However, the resistivity is generally between $100 \Omega-\mathrm{m}$ and $500 \Omega-\mathrm{m}$ typical of clayey sand (aquitard) i.e. a subsurface formation that stores but fairly transmit water. The best aquiferous zone is around north-eastern part, which is characterized by resistivity values in the range 200 to 700 $\Omega-\mathrm{m}$.

\section{Isopach Map of Overburden}

The isopach map of the overburden "Fig. 10" was obtained from the contour of the thickness of all the materials overlying the bedrock beneath the VES points against their geographic locations. The map shows overburden thickness varying from $0.8 \mathrm{~m}$ to $144.2 \mathrm{~m}$. The overburden thickness is generally greater than $20 \mathrm{~m}$ except the southeastern part which is characterized by thickness values less than $20 \mathrm{~m}$. The northeastern and southwestern parts of the institution show a high overburden thickness suggesting a high degree of weathering; which also implies that the basement rock is deep seated, while shallow in other parts of the institution.

\section{Isopach Map of the Fractured Basement}

The fractured basement forms one of the aquifers observed in the study area. The thickness distribution of the fractured basement is shown in "Fig. 11". The thickness varies between $0.8 \mathrm{~m}$ and $148 \mathrm{~m}$. The low thickness values in the range of $0 \mathrm{~m} 20 \mathrm{~m}$ trends in southwestern to northeastern direction, while the high thickness values (greater than $50 \mathrm{~m}$ ) characterized the northeastern to southwestern direction of the study area.

\subsection{Transverse Unit Resistance Map}

"Fig. 12" is the total transverse unit resistance map of the study area. The map shows that the transverse resistance is predominantly between 1000 and $10,000 \Omega \mathrm{m}^{2}$ indicated as medium groundwater development zone, this resistance values is found around the northwest through the central to the southeastern part of the study area. The high transverse resistance values (greater than $10,000 \Omega \mathrm{m}^{2}$ ) are found in northeastern and southwestern part of study area, these zones are the best area for groundwater development. However, pockets of low resistance values are observed around the southeastern part and central area of the study area. 
International Journal of Science and Research (IJSR)

ISSN (Online): 2319-7064

Index Copernicus Value (2013): 6.14 | Impact Factor (2014): 5.611

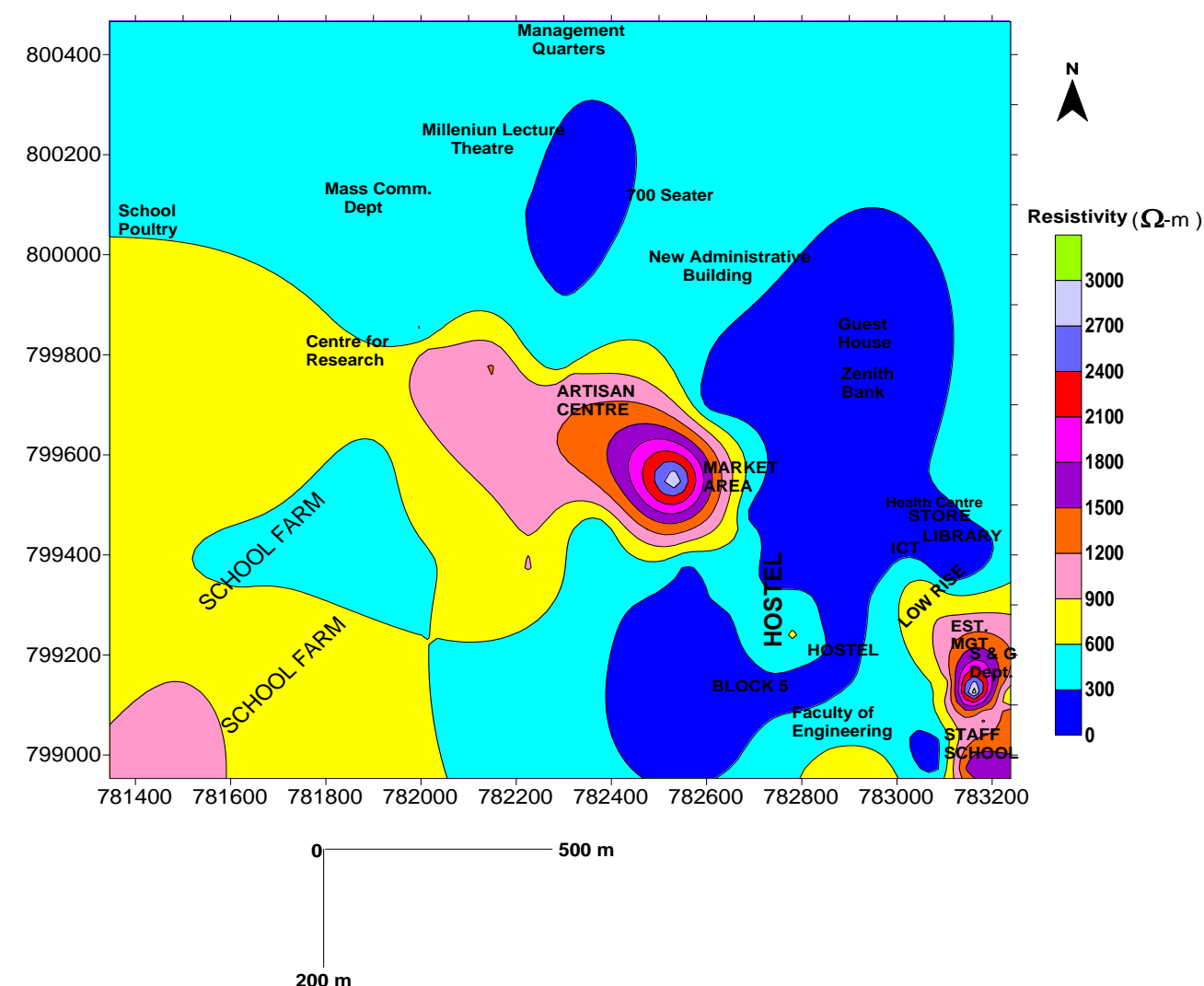

Figure 7: Isoresistivity Map of the Topsoil

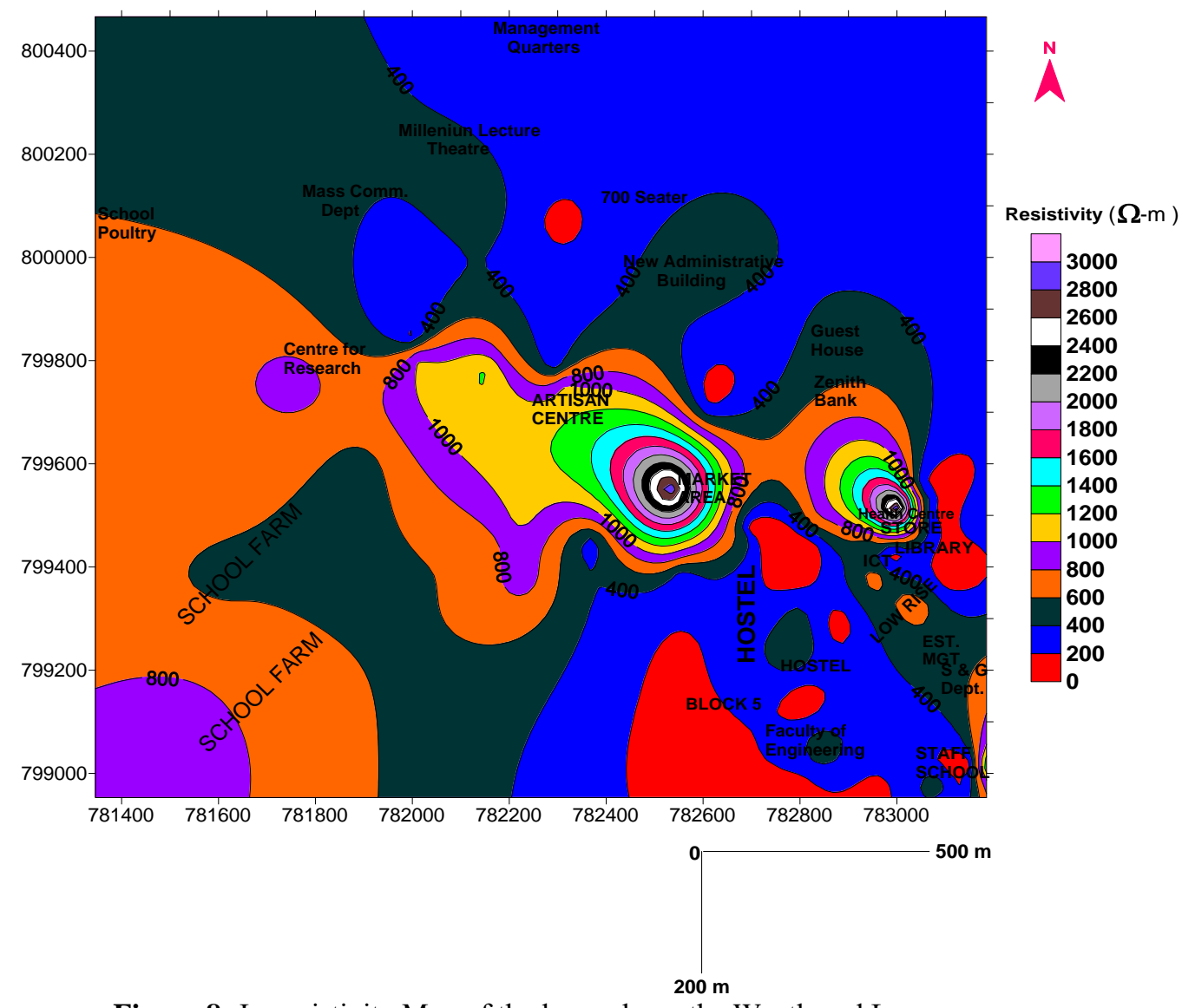

Figure 8: Isoresistivity Map of the layer above the Weathered Layer

Volume 4 Issue 12, December 2015

\author{
www.ijsr.net
}




\section{International Journal of Science and Research (IJSR) \\ ISSN (Online): 2319-7064}

Index Copernicus Value (2013): 6.14 | Impact Factor (2014): 5.611

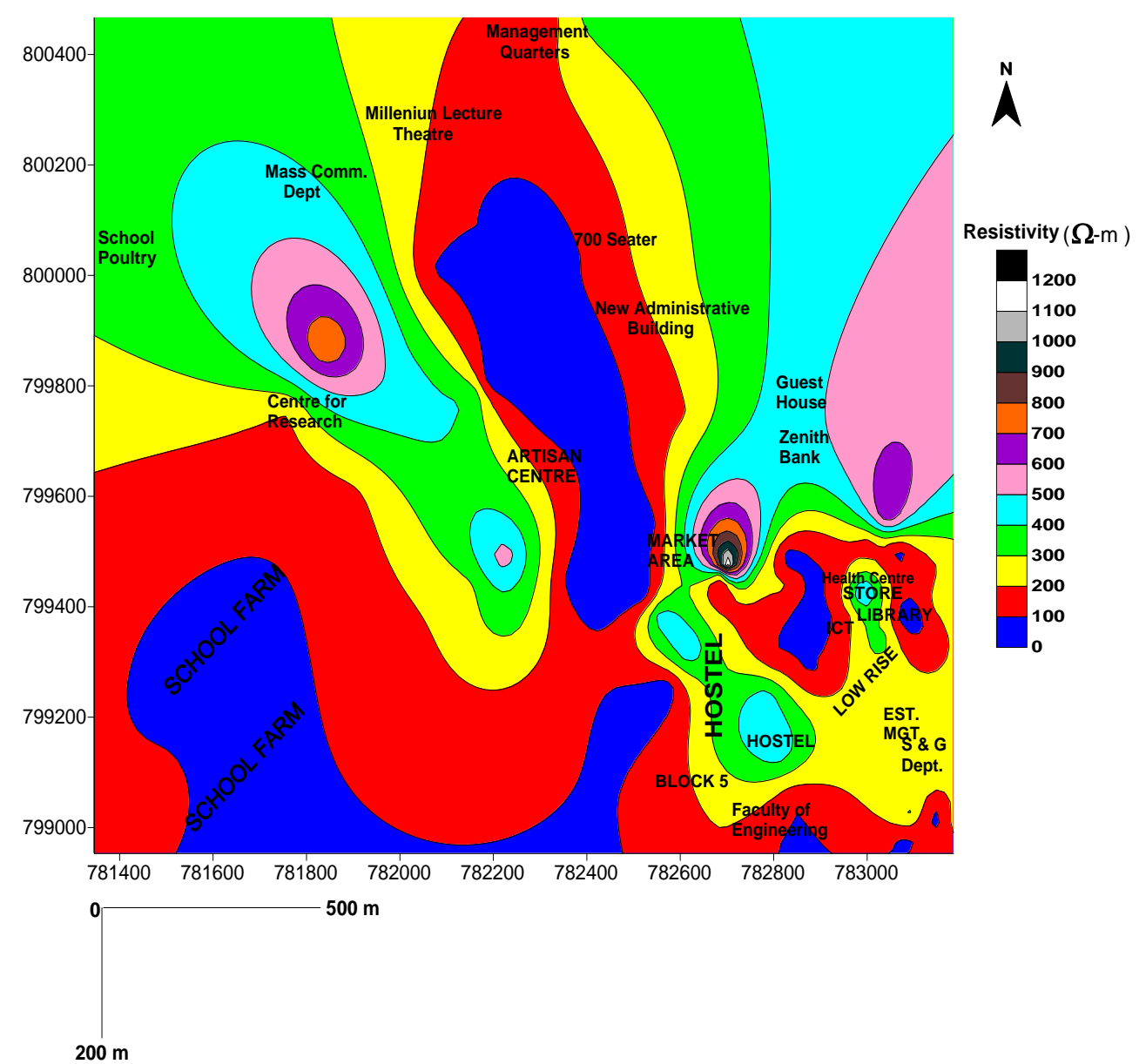

Figure 9: Isorestivity Map of the Weathered Layer

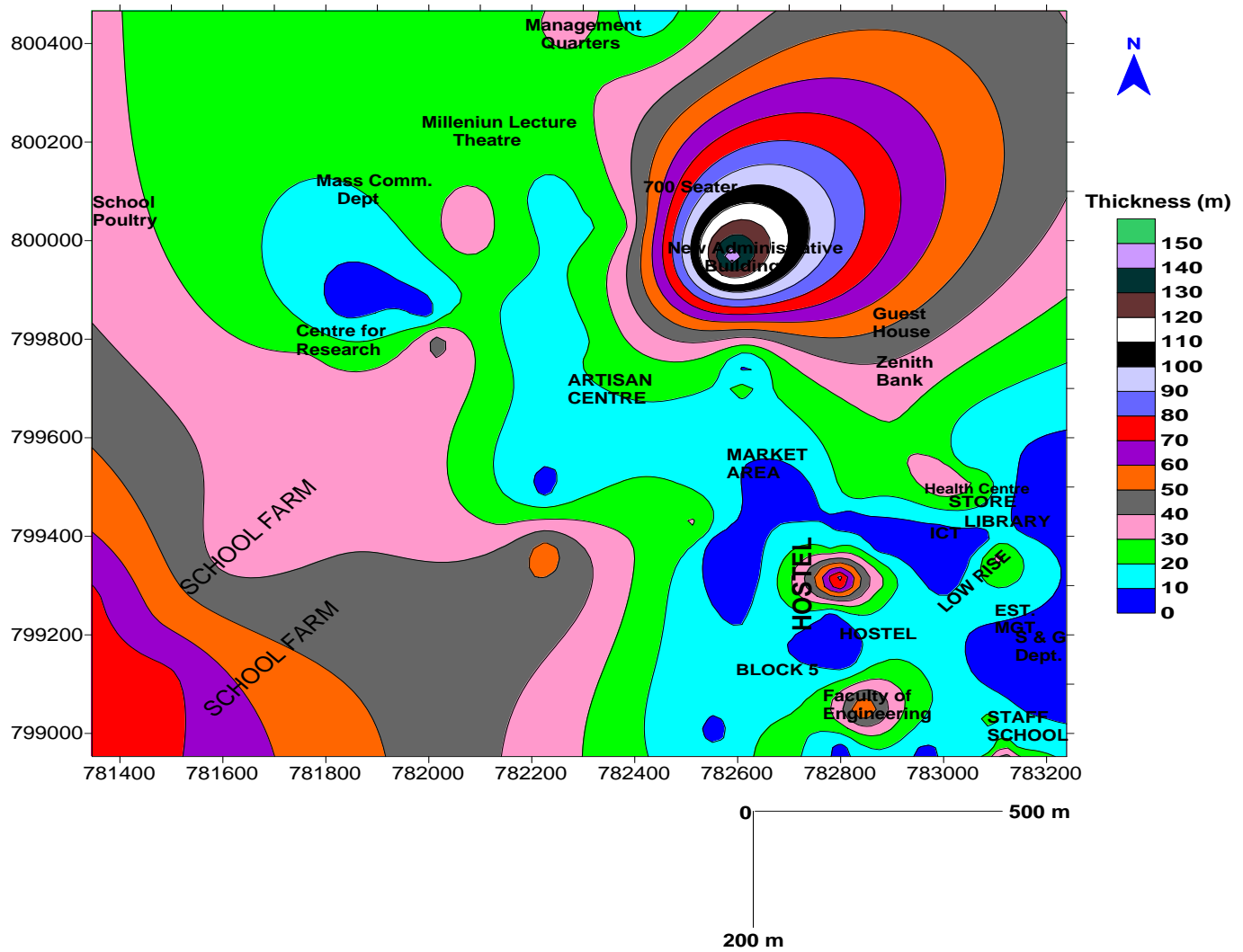

Figure 10: Isopach Map of the Overburden

Volume 4 Issue 12, December 2015

www.ijsr.net 
International Journal of Science and Research (IJSR)

ISSN (Online): 2319-7064

Index Copernicus Value (2013): 6.14 | Impact Factor (2014): 5.611

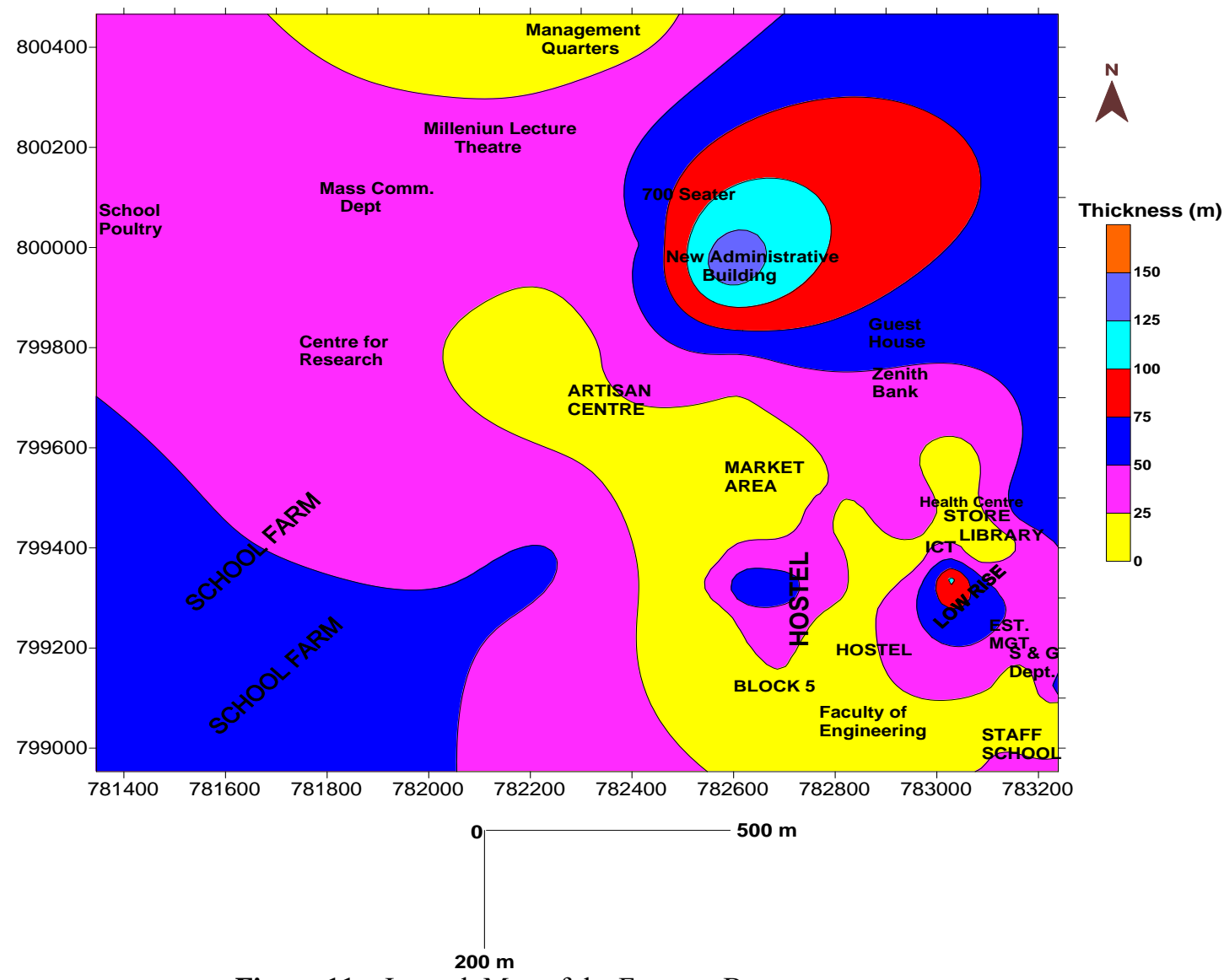

Figure 11: Isopach Map of the Fracture Basement

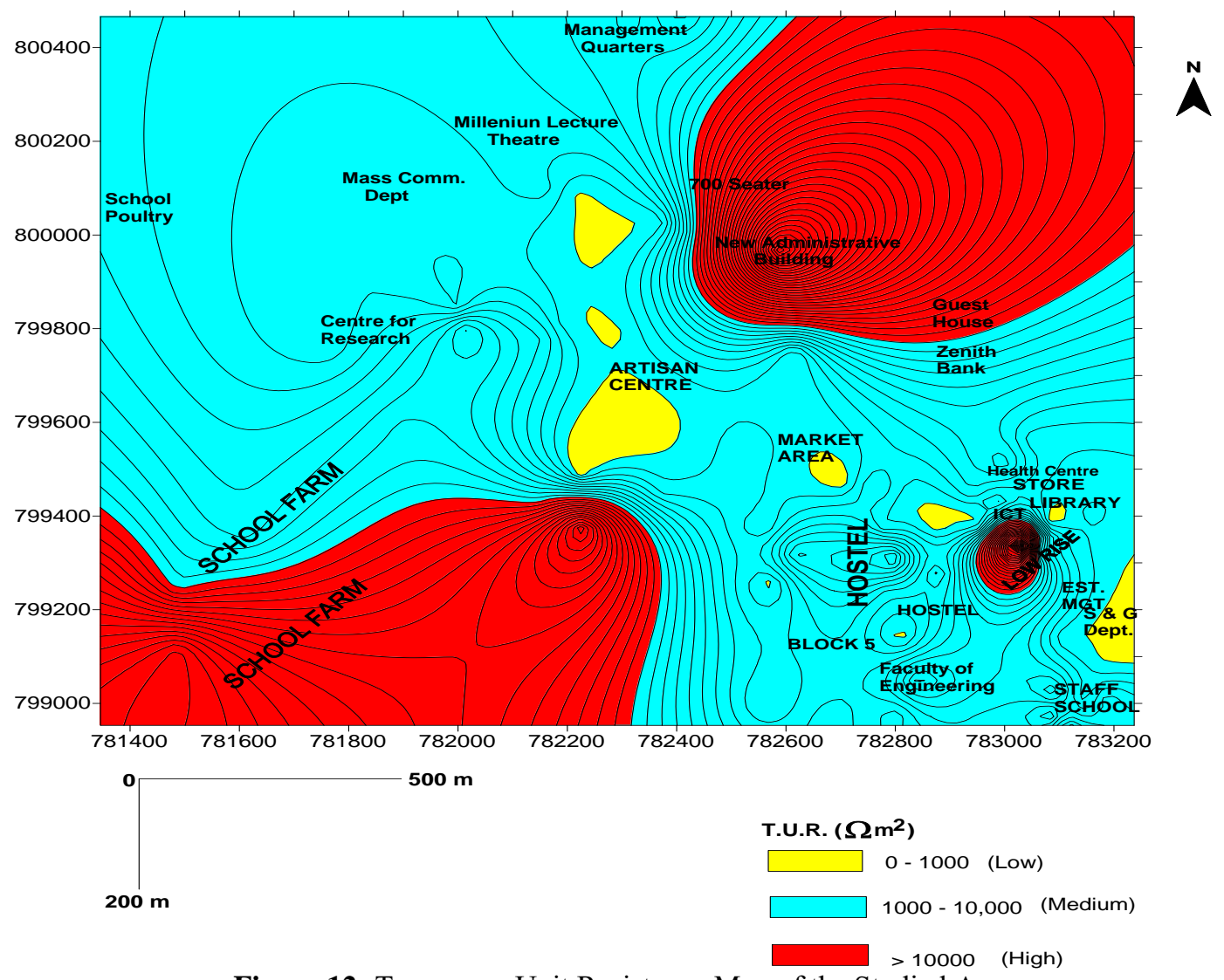

Figure 12: Transverse Unit Resistance Map of the Studied Area

Volume 4 Issue 12, December 2015

www.ijsr.net 


\section{International Journal of Science and Research (IJSR) \\ ISSN (Online): 2319-7064}

Index Copernicus Value (2013): 6.14 | Impact Factor (2014): 5.611

\subsection{Longitudinal Unit Conductance (L.U.C.) / Overburden Protective Capacity Evaluation}

The longitudinal unit conductance map "Fig. 13" shows the distribution of the longitudinal conductance within the study area. The map shows small closures of very high unit conductance $(>0.6$ mhos) in the southwestern, central and southeastern parts. Longitudinal unit conductances in the range of $0.15-0.5$ mhos is the most predominant in the study area.

Due to the filtering effect of the earth, the total longitudinal unit conductance values were used to evaluate the overburden protective capacity of the area, [12], [1]. [4] relate the protective capacity of an overburden overlying an aquifer to its hydraulic conductivity. The higher the clay content which impedes the fluid movement, characterized by low resistivity and low hydraulic conductivity, the higher the longitudinal unit conductance the better the protective capacity.

Table 2 shows the [4] longitudinal unit conductance classification as modified by [7] which is adopted in evaluating the protective capacity [1], [7], [12] in the study area. The overburden protective capacity map "Fig. 14" shows a moderate protective at the southwestern and northeastern/eastern parts, while all other areas have poor/weak protective capacity. A small closure of good protective capacity is observed in the eastern part.

The overburden protective map of the area shows that about $60 \%$ of the area falls within the moderate/good protective capacity, while $40 \%$ constitutes the poor/weak overburden protective rating. This suggests that the study area has weathering materials of good/moderate protective capacity.

\subsection{Coefficient of Anisotropy Map}

"Fig. 15" shows the coefficient of anisotropy map. This parameter describes the amount of subsurface layer anisotropy and generally lies in the range $1-2$ [14]. [10], [11] also revealed that the degree of subsurface inhomogeneity correlates linearly with groundwater yield.

The map shows that the coefficient of anisotropy values ranging from 0.16 to 33.8 units. The area is predominantly covered by low anisotropy coefficient $(<2.5)$ which indicates that the major aquifer present in the area is the weathered layer aquifer. Moderate anisotropy zone (2.5 - 5 units) is observed in the southeastern part, indicating presence of weathered/fractured basement aquifer. Closures of high anisotropic zones ( $>5$ unit) are observed within the moderate anisotropic zones; these zones suggest a highly fractured basement aquifer zones. It was also observed that most of the values $(85 \%)$ fall within the range of $1-2$ [14].

\subsection{Hydro-geological Measurements}

Measurement was taken on three (3) hand dug wells to reveal the depth of unsaturated zone (vadose water zone) and the water column. Tables 3 shows the data collected from hydrogeological measurement. The unsaturated zone (vadose) is the geologic materials between the earth's surface and the water table and can also be referred to as zone of aeration. The thickness of this zone determines the transit time, which is the time it will take for percolating water to reach the water table. It takes effluents one foot per year to migrate in the subsurface, therefore the thicker the vadose zone, the lesser the groundwater would be vulnerable to contaminants. This is because the effluents must have been filtered (attenuate) before reaching the aquifer.

The static water level, water column and depth to water level vary between $316.9 \mathrm{~m}$ and $330.5 \mathrm{~m}, 1.3 \mathrm{~m}$ and $4.8 \mathrm{~m}$, and 8.1 $\mathrm{m}$ and $10.5 \mathrm{~m}$ respectively. The average depth to the water level is $9.4 \mathrm{~m}$. This implies that the vadose zone is fairly thick; therefore the aquifer in the area would be less vulnerable to contamination. The average water column is $3.1 \mathrm{~m}$ indicating a medium water column.

\section{Conclusion}

Hydrogeologic studies and aquifer vulnerability studies of Rufus Giwa Polytechnic Owo has been carried out, which would provide a database for hydrogeological decisions like drill hole positioning or action plans for groundwater protection in the area. The findings of the study are:

1) The geoelectric sections generally revealed four (4) major layers comprising the topsoil, weathered layer, partly weathered/fractured basement, and fresh basement rock. The Weathered layer and unconfined basement are the two aquiferous zones observed in the area.

2)The geoelectrical maps show that best aquiferous zones with appreciable thickness are southwestern and northeastern parts of the area.

3)The average depth to the water level is $9.4 \mathrm{~m}$. This implies that the vadose zone is fairly thick; therefore the aquifer in the area would be less vulnerable to contamination. The average water column is $3.1 \mathrm{~m}$ indicating a medium water column.

4) The overburden protective map of the area shows that about $60 \%$ of the area falls within the moderate/good protective capacity, while $40 \%$ constitutes the poor/weak overburden protective rating. This suggests that the study area generally has weathering materials of good/moderate protective capacity.

The results of the study revealed that the aquifers within the study area are weathered layer and unconfined fractured basement. Considering the thickness of the vadose zone across the area, the resistivity parameters of the topsoil and the longitudinal conductance of the geomaterials, the best groundwater development areas with good/moderate protective capacity (from contaminants arising from anthropogenic activities) are northeastern and southwestern parts of the studied area. 


\section{International Journal of Science and Research (IJSR)}

ISSN (Online): 2319-7064

Index Copernicus Value (2013): 6.14 | Impact Factor (2014): 5.611

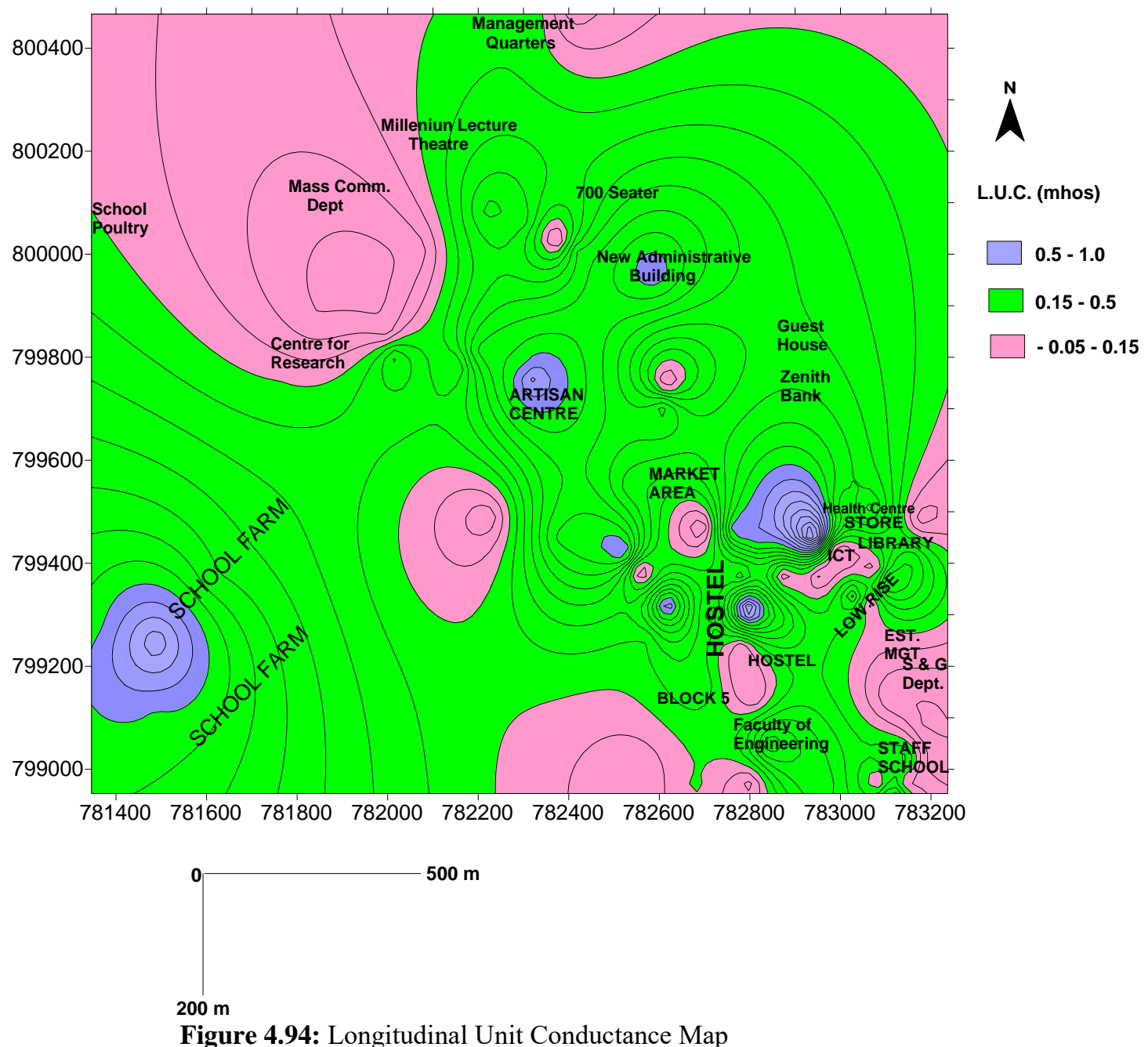

Table 2: Modified Longitudinal Conductance / Protective Capacity Rating

\begin{tabular}{|c|c|}
\hline Longitudinal Conductance (mhos) & Protective Capacity Rating \\
\hline$>10$ & Excellent \\
$5-10$ & Very Good \\
$0.7-4.9$ & Good \\
$0.2-0.69$ & Moderate \\
$0.1-0.19$ & Weak \\
$<0.1$ & Poor \\
\hline
\end{tabular}

\section{Recommendation}

1)Pre-drilling geophysical investigations including borehole logging should be conducted in the area to enhance or facilitate well design and completion processes for optimization of resulting borehole yield. This will prevent the common occurrence of borehole failure and lost of money.

2)Water quality analysis and complete borehole documentation should be carried out. This should be in accordance with known professional practice.

3) Groundwater monitoring should be conducted regularly in the area. 
International Journal of Science and Research (IJSR)

ISSN (Online): 2319-7064

Index Copernicus Value (2013): 6.14 | Impact Factor (2014): 5.611

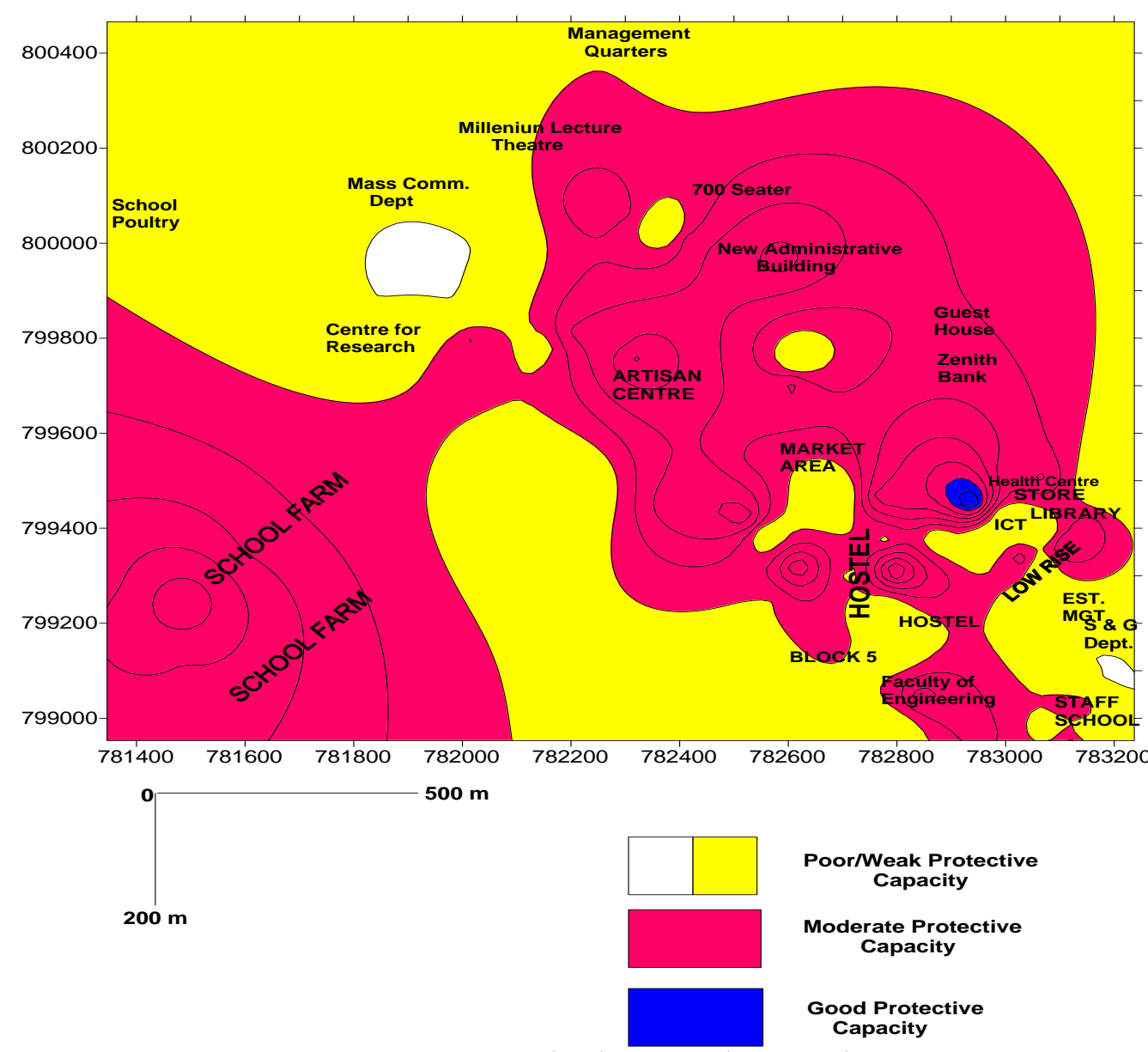

Figure 4.95: Overburden Protective Capacity Map

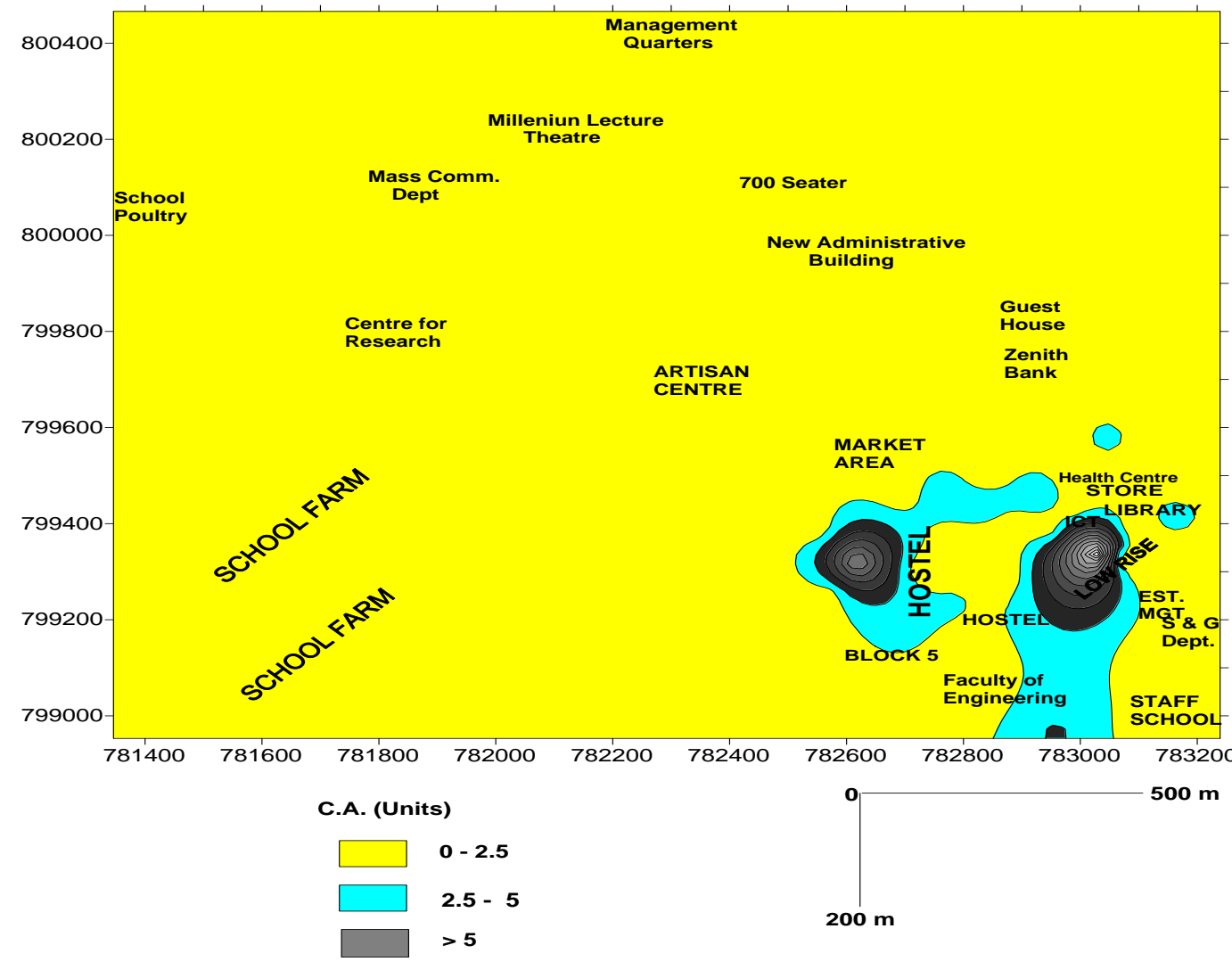

Figure 4.96: Coefficient of Anisotropy Map

Volume 4 Issue 12, December 2015

www.ijsr.net 


\section{International Journal of Science and Research (IJSR) \\ ISSN (Online): 2319-7064}

Index Copernicus Value (2013): 6.14 | Impact Factor (2014): 5.611

Table 3: Hydro-geological Field Data Obtained within the Study Area

\begin{tabular}{|c|c|c|c|c|c|c|c|}
\hline $\begin{array}{c}\text { Well No./ } \\
\text { Location }\end{array}$ & Easting & Northing & $\begin{array}{c}\text { Elevation } \\
(\mathrm{m})\end{array}$ & $\begin{array}{c}\text { Depth to Water Level } \\
(\mathrm{m})\end{array}$ & $\begin{array}{c}\text { Total Depth } \\
(\mathrm{m})\end{array}$ & $\begin{array}{c}\text { Water Column } \\
(\mathrm{m})\end{array}$ & $\begin{array}{c}\text { Static Water } \\
\text { Level }(\mathrm{m})\end{array}$ \\
\hline $1(\mathrm{SE})$ & 0781976 & 0799863 & 325 & 8.1 & 11.2 & 3.1 & 316.9 \\
\hline $2(\mathrm{SE})$ & 0783358 & 0799585 & 331 & 9.7 & 12.2 & \\
\hline $3(\mathrm{NE})$ & 0782782 & 0799244 & 341 & 10.5 & 15.3 & 3 & 4.8 \\
\hline
\end{tabular}

\section{References}

[1] O. Abiola, P.A. Enikanselu, M.I. Oladapo, "Groundwater potential and aquifer protective capacity of overburden units in Ado-Ekiti, Southwestern, Nigeria," International Journal of Physical Sciences, Vol. 4 (3), pp. 120-132, 2009.

[2] W.H. Barker, "Multispectral scanner. In: Janssen, L.L.F. and Huurneman, G.C. (eds), Principles of remote sensing, ITC Educational Textbook Series, pp. 71-82, 2001.

[3] R.W. Buddemeier, J.A Schloss, "Groundwater Storage and flow," http://www.kgs.Ukans.edu/Hight plains/atlas//apgngw.htm, 2000.

[4] J.P. Henriet, "Direct Application of the Dar Zarrock parameters in groundwater surveys," Geophysical Prospecting, Vol 24, pp. 344-356, 1976.

[5] N.P. Iloeje, A new geography of Nigeria (New Revised Edition) published by Longman Nig. Ltd., Lagos, $201 \mathrm{pp}, 1981$.

[6] M.J. Jones, "The weathered zone aquifers of Basement Complex area of Africa," Q.J. Eng. Geol. London, Vol. 18 , pp. $35-46,1985$

[7] M.I. Oladapo, O.J. Akintorinwa, "Hydrogeophysical Study of Ogbese, Southwestern, Nigeria,” Global J. Pure and Applied Sci. 13(1): 55-61, 2007.

[8] V.O. Olarewaju, "Charnockite-Granite Association in S.W. Nigeria: Rapakivi granite type and charnockitic plutonism in Nigeria," J.Afri. Earth Sci., 6, No. 1. Pp 67-77, 1987.

[9] M.O. Olorunfemi, S.A.Fasuyi, "Aquifer types and the geoelectric/hydrogeological characteristics of part of the central basement terrain of Nigeria (Niger State)," Journal of African Earth Science: 16, 309-317, 1993.

[10]M.O.Olorunfemi, V.O. Olarewaju, O. Alade, "On the electrical anisotropic and groundwater yield in a basement complex area of S.W. Nigeria," Journal of African Earth Science, Vol. 12, No. 3, pp. 462-472, 1991.

[11]M.O. Olorunfemi, "Voyage on the Skin of the Earth: A geophysical experience Inaugural Lecture Series 211, Obafemi Awolowo University, Ile-Ife, pp. 23-30, 2008.

[12]K.K. Onifade, "Geoelectric Investigation of Groundwater Potential and Aquifer Vulnerability of Ore, Southwestern Nigeria. Unpub. M.Tech. Thesis, Federal University of Technology, Akure, Nigeria. 67pp, 2008.

[13]E. Orellana, H.M. Mooney, "Master Tables and Curves for Vertical Electrical Sounding over Layered Structure: Geophy, Prospect," 29: 932-955, 1966.

[14]J.M. Reynolds, "An Introduction to Applied and Environmental Geophysics," Published by John Wiley and Sons Ltd, England, pp 212-320, 1997.

[15]B.N. Satpathy, D.N. Kanungo, "Water exploration on land trerrain: A case history," Geophysical Prospecting, 24: 725-736, 1976.
[16]B.P.A.Vander Velper, "Resist Version 1.0," M.Sc. research Project ITC Delf. Netherlands, 1988.

\section{Author Profile}

Falowo Olumuyiwa Olusola has an M.Tech. degree and M.Phil. in Exploration Geophysics and Hydrogeology/Environmental Geology respectively. He is a postgraduate student of Geology Department in University of Benin, Edo State, Nigeria.

Imeokparia Ebo Gabriel is a professor of Applied Geology in Department of Geology, University of Benin, Nigeria. His research areas are Exploration Geology/Geochemistry, Environmental Geochemistry, Ore Deposit Studies and Petrology. 